\title{
Orbital Reversibility of Planar Vector Fields
}

\author{
Antonio Algaba ${ }^{1}$, Cristóbal García ${ }^{1}$ (D) and Jaume Giné ${ }^{2, *}$ (D) \\ 1 Centro de Estudios Avanzados en Física, Departament Ciencias Integradas, Matemáticas y Computación, \\ Facultad de Ciencias, University of Huelva, 21007 Huelva, Spain; algaba@uhu.es (A.A.); \\ cristoba@uhu.es (C.G.) \\ 2 Inspires Research Centre, Departament de Matemàtica, Universitat de Lleida, Av. Jaume II, 69, \\ 25001 Lleida, Spain \\ * Correspondence: gine@matematica.udl.cat
}

check for updates

Citation: Algaba, A.; García, C.; Giné, J. Orbital Reversibility of Planar Vector Fields. Mathematics 2021, 9, 14. https://dx.doi.org/10.3390/math90100 14

Received: 29 August 2020

Accepted: 15 October 2020

Published: 23 December 2020

Publisher's Note: MDPI stays neutral with regard to jurisdictional claims in published maps and institutional affiliations.

Copyright: () 2020 by the authors. Licensee MDPI, Basel, Switzerland. This article is an open access article distributed under the terms and conditions of the Creative Commons Attribution (CC BY) license (https: / creativecommons.org/ licenses/by/4.0/).

\begin{abstract}
In this work we use the normal form theory to establish an algorithm to determine if a planar vector field is orbitally reversible. In previous works only algorithms to determine the reversibility and conjugate reversibility have been given. The procedure is useful in the center problem because any nondegenerate and nilpotent center is orbitally reversible. Moreover, using this algorithm is possible to find degenerate centers which are orbitally reversible.
\end{abstract}

Keywords: time-reversibility; orbital reversibility; center problem; planar vector fields

\section{Introduction}

In this work we consider vector fields in $\mathbb{R}^{2}$. A vector field $\mathbf{F}$ is called reversible if there is an involution $\sigma$ defined on the plane that fixes an analytic curve passing through the origin and transforms $\mathbf{F}$ into $-\mathbf{F}$. The most important involutions are $\sigma(x, y)=(-x, y)$ and $\sigma(x, y)=(x,-y)$ because any other involution (linear or not) can be transformed in these ones as is explained later. Vector fields reversible under these involutions are called, respectively, $R_{x}$-reversible and $R_{y}$-reversible and, in general, time-reversible vector fields. Orbital reversibility requires reversibility with respect to an involution and a spacedependent change of the temporal variable. The main result of the paper is to determine when a planar vector field is orbitally reversible proving the existence of a normal form in which some higher-order terms must vanish (those that obstruct the orbital-reversibility).

Any invariance of a differential equation is always associated with a symmetry of such differential equation. The most studied symmetries are the time-reversal symmetries that, for a differential system in the plane, corresponds to a specular symmetry with respect to one axis of symmetry. In fact a time-reversal symmetry is one of the fundamental symmetries that appears in nature, see for instance [1-6] and references therein. The timereversal symmetry arises in many physically motivated systems both in classical and quantum mechanics. For instance, a simple example is to consider a pendulum swing without friction. If we see the pendulum moving backward in time the motion also corresponds to a possible movement of the pendulum. Hence the system has a timereversal symmetry. However the presence of friction breaks the time-reversal symmetry we can distinguish between a motion forward or backward in time. It is clear that a swinging pendulum cannot increase its amplitude in time unless there is a source of energy feeding the pendulum. Nevertheless what was unknown until now, which is solved in this work, is how to determine if a differential system in the plane has a time-reversal symmetry. Notwithstanding in statistical mechanics, when we describe a dynamical system of large number of particles the time-reversal symmetry is broken and there is a true sense of direction in time. In this context we have Boltzmann's second law of thermodynamics, saying that entropy is always a monotonically increasing function of time. This is not in contradiction with the movement of each particle of the system that can have time-reversal symmetry because the more probabilistic global movement implies the irreversibility of 
time. In fact, in a system that can have a time-reversal symmetry on a microscopic scale, this symmetry is broken in its collective macroscopic behavior. A very effective example of the irreversibility of a macroscopic process is the exit of a gas from a bottle when the stopper is removed. This is an irreversible process since we will never see the molecules of the gas returning spontaneously into the bottle although the movement of each molecule is reversible.

The algorithm presented here is important because it allows to determine if there exist a change of coordinates and a scaling of time (orbital reversibility) in which the system has a time-reversal symmetry. Remark that, usually, the existence of such symmetries is hidden. Hence a differential system is orbitally reversible if there is a formal change of variables and a change of time such that the transformed system is reversible.

The axis of symmetry is, in the general case, an analytic curve. However, from the work of Montgomery and Zippin [7], any analytic involution associated with a timereversal symmetry can be linearized in such a way that the symmetry axis becomes a straight line. Furthermore, by a rotation, the symmetry axis can become the $x$ - or $y$-axis. At the beginning of the last century Birkhoff considered reversible vector fields to study the restricted three-body problem. Some decades later the theory of reversible vector fields was formalized by Devaney [8].

There is a narrow relation between the reversibility and the center problem. We recall here that the center problem seeks to distinguish between a focus or a center in a monodromic planar differential system, see [9-17]. It is well-known that an analytic system of differential equations having a nondegenerate center is a reversible system with respect to a nonlinear analytic involution (see [12,18-21]) and a nilpotent center is always an orbitally reversible system (see $[11,12,22,23]$ ). Nevertheless there are degenerate centers of analytic differential systems which are not orbitally reversible systems, see [24].

In any case, the relation between reversibility and degenerate centers can be established with weaker conditions. For instance, in [25] it is proved the existence of a smooth map that transforms an analytic system having a degenerate center at the origin (with either an analytic first integral or a smooth inverse integrating factor) into a reversible linear system (after a rescaling of time). Moreover in [26] it is proved that for any degenerate center singular point of an analytic planar system always admits a smooth inverse integrating factor in a neighborhood of it. The loss of analyticity of the inverse integrating factor does not allow to construct an algorithm to determine necessary conditions to have a degenerate center and consequently this remains as an open problem, see [27,28]. In [13,14,29-31], reversibility and its relationship with the center problem is studied. In fact very few mechanisms for producing centers are known; the analytic integrability, the existence of a particular integrating factor and the orbitally reversibility are the most common. In this work we give an algorithm to determine the last sufficient condition to have a center.

Algorithms to determine the time-reversibility and conjugate reversibility (they do not include the time reparametrization) have been given in [32-34]. Moreover, in [35] the orbitally reversibility is studied for a particular class of planar dynamical systems. In [36] the center conditions of a particular case of polynomial nilpotent system are studied using an orbital reversible algorithm for such particular systems. Generalizing these works here we give an algorithm to determine if any differential system in the plane is orbitally reversible. In fact, we construct a unique normal form that determines the invariants that prevent the orbital reversibility of the vector field studied.

The main results of the present paper are given in Theorem 2 given in Section 3 and Theorem 4 given in Section 4 . Theorems 2 and 4 provide necessary and sufficient conditions of orbital $R_{x}$ - and $R_{y}$-reversibility, respectively. In the next section some preliminary definitions and results necessary to prove the main results are given. The work finishes with a section of applications of the algorithm provided. 


\section{Definitions and Preliminary Results}

This paper deals with analytic differential systems of the form

$$
\dot{\mathbf{x}}=\mathbf{F}(\mathbf{x}), \mathbf{x} \in \mathbb{R}^{2},
$$

having an equilibrium point located at the origin. We address the orbital-reversibility problem, which consists in determining if system (1) admits some reversibility (possibly nonlinear) modulo formal equivalence. In the paper, we will consider only involutions having fixed point set with codimension one. Our aim is to adapt the ideas of the normal form theory under equivalence to characterize orbital-reversibility in planar vector fields. First we introduce some definitions and terminology, related to the formal normal form theory in the quasi-homogeneous context. Our analysis is based in an adequate classification of system (1), obtained by using nonlinear time-reparametrizations and coordinate transformations.

Let us consider a time-reparametrization $d t / d T=1+\mu(\mathbf{x})$, with $\mu(\mathbf{0})=0$. Then, it is easy to show that the vector field $\mathbf{F}$ of $(1)$ is transformed into $(1+\mu) \mathbf{F}$.

More involved is the analysis of the effect of transformations in the state variables. We denote the transformed vector field of $\mathbf{F}$ by a transformation $\Phi$ as $\Phi{ }_{*} \mathbf{F}$. In our study, it is more convenient to use the generator of the transformation, which defines a Cauchy problem whose solution is the transformation. Namely, any near-identity transformation $\mathbf{y}=\Phi(\mathbf{x})$ is associated to a generator $\mathbf{U}(\mathbf{x})$ (see [37]) in such away that

$$
\Phi(\mathbf{x})=\mathbf{x}+\mathbf{U}(\mathbf{x})+\frac{1}{2 !} D \mathbf{U}(\mathbf{x}) \mathbf{U}(\mathbf{x})+\cdots .
$$

If we use a generator $\mathbf{U}$ corresponding to the transformation $\Phi$, the transformed vector field will be denoted as $\mathbf{U}_{* *} \mathbf{F}:=\Phi_{*} \mathbf{F}$.

The basic tool in the characterization of the transformed vector field is the Lie product, defined by

$$
[\mathbf{F}, \mathbf{U}](\mathbf{x})=D \mathbf{F}(\mathbf{x}) \mathbf{U}(\mathbf{x})-D \mathbf{U}(\mathbf{x}) \mathbf{F}(\mathbf{x}),
$$

where $\mathbf{F}, \mathbf{U}$ are smooth vector fields. The transformed system can be efficiently expressed in terms of nested Lie products as follows. Let us define $T_{\mathbf{U}}^{(0)}(\mathbf{F}):=\mathbf{F}$, and

$$
T_{\mathbf{U}}^{(l)}(\mathbf{F}):=T_{\mathbf{U}}^{(l-1)}([\mathbf{F}, \mathbf{U}])=\overbrace{[\cdots[}^{l \text { times }} \mathbf{F}, \mathbf{U}], \cdots, \mathbf{U}]=\left[T_{\mathbf{U}}^{(l-1)}(\mathbf{F}), \mathbf{U}\right], \text { for } l \geq 1 .
$$

Then, we can write the transformed system as (see [37])

$$
\mathbf{U}_{* *} \mathbf{F}:=\sum_{l=0}^{\infty} \frac{1}{l !} T_{\mathbf{U}}^{(l)}(\mathbf{F})=\mathbf{F}+[\mathbf{F}, \mathbf{U}]+\frac{1}{2 !}[[\mathbf{F}, \mathbf{U}], \mathbf{U}]+\cdots .
$$

In summary, if we use both, a nonlinear time-reparametrization $d t=(1+\mu(\mathbf{x})) d T$ and a near-identity transformation with generator $\mathbf{U}(\mathbf{x})$, the vector field transformed of $\mathbf{F}$ by means of the change of variables with generators (spatial $\mathbf{U}$ and temporal $1+\mu$ ) is given by

$$
\mathbf{U}_{* *}((1+\mu) \mathbf{F})=(1+\mu) \mathbf{F}+[(1+\mu) \mathbf{F}, \mathbf{U}]+\frac{1}{2 !}[[(1+\mu) \mathbf{F}, \mathbf{U}], \mathbf{U}]+\cdots .
$$

In the classical normal form theory, the analysis of simplifications in the vector field $\mathbf{F}$ is done by using a linear transformation and a sequence of near-identity transformations of successive degrees through its Taylor expansions. More concretely, the linear part requires linear changes that are not of the near-identity type.

We will use here a more general perspective by means of quasi-homogeneous expansions for the vector fields. 
We now introduce some notation in order to work in the quasi-homogeneous frame. A scalar polynomial $f$ is quasi-homogeneous of type $\mathbf{t}=\left(t_{1}, t_{2}\right) \in \mathbb{N}^{2}$ and degree $k$ if $f\left(\varepsilon^{t_{1}} x, \varepsilon^{t_{2}} y\right)=\varepsilon^{k} f(x, y)$. The vector space of quasi-homogeneous scalar polynomials of type $\mathbf{t}$ and degree $k$ is denoted by $\mathcal{P}_{k}^{\mathbf{t}}$. A polynomial vector field $\mathbf{F}=(P, Q)^{T}$ is quasihomogeneous of type $\mathbf{t}$ and degree $k$ if $P \in \mathcal{P}_{k+t_{1}}^{\mathbf{t}}$ and $Q \in \mathcal{P}_{k+t_{2}}^{\mathbf{t}}$. The vector space of polynomial quasi-homogeneous vector fields of type $\mathbf{t}$ and degree $k$ is denoted by $\mathcal{Q}_{k}^{\mathbf{t}}$. Given an analytic vector field $\mathbf{F}$, we can write it as a quasi-homogeneous expansion corresponding to a fixed type $\mathbf{t}$ :

$$
\mathbf{F}(\mathbf{x})=\mathbf{F}_{r}(\mathbf{x})+\mathbf{F}_{r+1}(\mathbf{x})+\cdots=\sum_{j \geq r} \mathbf{F}_{j}(\mathbf{x}),
$$

where $\mathbf{x} \in \mathbb{R}^{2}, r \in \mathbb{Z}$ and $\mathbf{F}_{j} \in \mathcal{Q}_{j}^{\mathbf{t}}$, i.e., each term $\mathbf{F}_{j}$ is a quasi-homogeneous vector field of type $\mathbf{t}$ and degree $j$.

We now need to introduce some definitions and terminology of the normal forms. We denote the operator lineal $\ell_{k}$ (the Lie-derivative operator of $\mathbf{F}_{r}$ ) as

$$
\begin{aligned}
\ell_{k}: \mathcal{P}_{k-r}^{\mathbf{t}} & \longrightarrow \mathcal{P}_{k}^{\mathbf{t}} \\
\mu_{k-r} & \rightarrow \nabla \mu_{k-r} \cdot \mathbf{F}_{r} .
\end{aligned}
$$

An involution is a formal diffeomorphism $\sigma$, such that $\sigma \circ \sigma=I d$. Denote Fix $(\sigma)=$ $\{\mathbf{x} \mid \sigma(\mathbf{x})=\mathbf{x}\}$. This set is a local sub-manifold of $\mathbb{R}^{2}$ and we are assuming throughout the paper that $\operatorname{dim}(\operatorname{Fix}(\sigma))=1$.

We say that the system $\dot{\mathbf{x}}=\mathbf{F}(\mathbf{x}), \mathbf{x} \in \mathbb{R}^{2}$, or the vector field $\mathbf{F}$ is reversible if there is an involution $\sigma, \sigma(\mathbf{0})=\mathbf{0}$, such that $\sigma_{*} \mathbf{F}=-\mathbf{F}$.

We say that the system $\dot{\mathbf{x}}=\mathbf{F}(\mathbf{x}), \mathbf{x} \in \mathbb{R}^{2}$, or the vector field $\mathbf{F}$ is orbitally reversible if there exists an involution $\sigma$ and a formal function $\mu, \mu(\mathbf{0})=0$ such that $\sigma_{*}((1+\mu) \mathbf{F})=$ $-(1+\mu) \mathbf{F}$.

We say that the system $\dot{\mathbf{x}}=\mathbf{F}(\mathbf{x})$, or the vector field $\mathbf{F}$ is reversible with respect to the coordinate $x(y)$ or just $R_{x}$-reversible $\left(R_{y}\right.$-reversible), if it is reversible with respect to the involution

$$
\sigma(x, y)=(-x, y), \quad(\sigma(x, y)=(x,-y)) .
$$

We mean that the system $\dot{\mathbf{x}}=\mathbf{F}(\mathbf{x})$ is invariant under the symmetry given by $(x, y, t) \rightarrow$ $(-x, y,-t)$ or $(x, y, t) \rightarrow(x,-y,-t)$, respectively. In turn, this implies, for instance in the second case, that when $(x(t), y(t))$ is a trajectory in phase space describing a possible motion of the system with initial position and momentum $\left(x_{0}, y_{0}\right)$, then so is $(x(-t),-y(-t))$ with initial condition $\left(x_{0},-y_{0}\right)$. In configuration (position) space this means that if we have a trajectory $x(t)$, then we also have a trajectory $x(-t)$. This is precisely what we see when we observe a time-reversible system in reverse time.

The following result provides a necessary and sufficient condition for vector field (2) to be orbitally reversible. This result is an adaptation of [32] [Theorem 2.3 and Corollary 1] and [35] [Theorem 2.3], in this case, using spatial and temporal generators.

Theorem 1. The vector field $\mathbf{F}$, given in (2), is orbital reversible if, and only if, there exists $\mathbf{U}$ (generator of a change of variable close to the identity), a scalar function $\mu$ with $\mu(\mathbf{0})=0$ and $\Phi_{0} \in \mathcal{Q}_{0}^{\mathbf{t}}$ such that $\mathbf{U}_{* *}\left((1+\mu)\left(\Phi_{0}\right)_{*} \mathbf{F}\right)$ is axis-reversible (i.e., $R_{x}$ or $R_{y}$-reversible).

Notice that in order to be $\mathbf{F}$ orbital reversible it is necessary that there exists $\Phi_{0} \in \mathcal{Q}_{0}^{\mathbf{t}}$ such that $\left(\Phi_{0}\right)_{*} \mathbf{F}_{r}$ be $R_{x}$ - or $R_{y}$-reversible. Thus, without loss of generality (applying a change of variables of degree $0, \Phi_{0}$, given by the previous Proposition), we can consider $\left(\Phi_{0}\right)_{*} \mathbf{F}$ instead of $\mathbf{F}$ and, in this way, we can start from the vector field

$$
\mathbf{F}=\widetilde{\mathbf{F}}_{r}+\sum_{j>r} \mathbf{F}_{j}
$$

where $\widetilde{\mathbf{F}}_{r}$ is $R_{x}$-reversible. (The $R_{y}$-reversible case is treated analogously). 
Notice that $\widetilde{\mathbf{F}}_{r} \in \mathcal{Q}_{j}^{\mathbf{t}}$ can be uniquely written as

$$
\widetilde{\mathbf{F}}_{r}=\mathbf{X}_{h}+\mu \mathbf{D}_{0}
$$

where $\mu:=\frac{1}{r+|\mathbf{t}|} \operatorname{div} \widetilde{\mathbf{F}}_{r} \in \mathcal{P}_{r}^{\mathbf{t}}, h:=\frac{1}{r+|\mathbf{t}|} \mathbf{D}_{0} \wedge \widetilde{\mathbf{F}}_{r} \in \mathcal{P}_{r+|\mathbf{t}|}^{\mathbf{t}},|\mathbf{t}|:=t_{1}+t_{2}, \mathbf{D}_{0}:=\left(t_{1} x, t_{2} y\right)^{T}$, and $\mathbf{X}_{h}:=(-\partial h / \partial y, \partial h / \partial x)^{T}$ is the Hamiltonian vector field with Hamiltonian function $h$, see [38] [Prop.2.7].

In order to study the orbital $R_{x}$-reversibility of the vector field (3), we have to construct a suitable normal form (suitable spatial and temporal generators) that allows us to distinguish the terms that obstruct the orbital $R_{x}$-reversibility of the vector field studied. To study this problem and state our main result we need to define some subspaces that will constitute the initial and final space of the homological operators that will define the adequate normal form to study the problem of orbital reversibility. We need the following subspaces:

$$
\begin{aligned}
& \widetilde{\mathcal{P}}_{k}^{\mathrm{t}}:=\left\{\mu \in \mathcal{P}_{k}^{\mathrm{t}}: \mu(-x, y)=-\mu(x, y)\right\}, \text { (odd scalar function) } \\
& \overline{\mathcal{P}}_{k}^{\mathrm{t}}:=\left\{\mu \in \mathcal{P}_{k}^{\mathrm{t}}: \mu(-x, y)=\mu(x, y)\right\} \text { (even scalar function) } \\
& \widetilde{\mathcal{Q}}_{k}^{\mathrm{t}}:=\left\{(P, Q)^{T} \in \mathcal{Q}_{k}^{\mathrm{t}}: P \in \overline{\mathcal{P}}_{k+t_{1}}^{\mathrm{t}}, Q \in \widetilde{\mathcal{P}}_{k+t_{2}}^{\mathrm{t}}\right\}, \text { (vector field reversible to } x \text { ) } \\
& \overline{\mathcal{Q}}_{k}^{\mathrm{t}}:=\left\{(P, Q)^{T} \in \mathcal{Q}_{k}^{\mathrm{t}}: P \in \widetilde{\mathcal{P}}_{k+t_{1}}^{\mathrm{t}}, Q \in \overline{\mathcal{P}}_{k+t_{2}}^{\mathrm{t}}\right\}, \text { (symmetric vector field) }
\end{aligned}
$$

It is verified that $\mathcal{Q}_{k}^{\mathbf{t}}=\widetilde{\mathcal{Q}}_{k}^{\mathbf{t}} \oplus \overline{\mathcal{Q}}_{k}^{\mathbf{t}}$ and $\mathcal{P}_{k}^{\mathbf{t}}=\widetilde{\mathcal{P}}_{k}^{\mathbf{t}} \oplus \overline{\mathcal{P}}_{k}^{\mathbf{t}}$.

Let $\mathbf{P}_{k} \in \mathcal{Q}_{k^{\prime}}^{\mathrm{t}} \mu_{k} \in \mathcal{P}_{k^{\prime}}^{\mathrm{t}}$ we denote $\widetilde{\mathbf{P}}_{k}=\operatorname{Proy}_{\widetilde{\mathcal{Q}}_{k}^{\mathrm{t}}}\left(\mathbf{P}_{k}\right), \overline{\mathbf{P}}_{k}=\operatorname{Proy}_{\overline{\mathcal{Q}}_{k}^{\mathrm{t}}}\left(\mathbf{P}_{k}\right), \widetilde{\mu}_{k}=$ $\operatorname{Proy}_{\widetilde{\mathcal{P}}_{k}^{\mathrm{t}}}\left(\mu_{k}\right)$ and $\bar{\mu}_{k}=\operatorname{Proy}_{\overline{\mathcal{P}}_{k}^{\mathrm{t}}}\left(\mu_{k}\right)$. We will use the same notation for the case of sum of quasi-homogeneous fields or scalar functions.

The following result shows how the bracket and Lie derivative behave when taking vector fields and scalar functions with symmetries.

Lemma 1. Let $\widetilde{\mathbf{F}}_{r} \in \widetilde{\mathcal{Q}}_{r}^{\mathbf{t}}, \overline{\mathbf{F}}_{s} \in \overline{\mathcal{Q}}_{s}^{\mathbf{t}}, \widetilde{\mu}_{k} \in \widetilde{\mathcal{P}}_{k^{\prime}}^{\mathbf{t}} \bar{\mu}_{k^{\prime}}^{\mathbf{t}} \widetilde{\mathbf{P}}_{k} \in \widetilde{\mathcal{Q}}_{k^{\prime}}^{\mathbf{t}} \overline{\mathbf{P}}_{k}^{\mathbf{t}} \in \overline{\mathcal{Q}}_{k}^{\mathbf{t}}$. Then
a) $\widetilde{\mu}_{k} \widetilde{\mu}_{s} \in \overline{\mathcal{P}}_{k+s}^{\mathrm{t}}$
b) $\widetilde{\mu}_{k} \bar{\mu}_{s} \in \widetilde{\mathcal{P}}_{k+s}^{\mathbf{t}}$
c) $\bar{\mu}_{k} \bar{\mu}_{s} \in \overline{\mathcal{P}}_{k+r}^{\mathrm{t}}$
d) $\widetilde{\mu}_{k} \cdot \widetilde{\mathbf{F}}_{r} \in \overline{\mathcal{Q}}_{k+r}^{\mathrm{t}}$
e) $\widetilde{\mu}_{k} \cdot \overline{\mathbf{F}}_{s} \in \widetilde{\mathcal{Q}}_{k+s}^{\mathbf{t}}$
f) $\bar{\mu}_{k} \cdot \widetilde{\mathbf{F}}_{r} \in \widetilde{\mathcal{Q}}_{k+r}^{\mathbf{t}}$
g) $\bar{\mu}_{k} \cdot \overline{\mathbf{F}}_{s} \in \overline{\mathcal{Q}}_{k+r}^{\mathrm{t}}$
h) $\nabla \widetilde{\mu}_{k} \cdot \widetilde{\mathbf{F}}_{r} \in \overline{\mathcal{P}}_{k+r}^{\mathbf{t}}$
i) $\nabla \widetilde{\mu}_{k} \cdot \overline{\mathbf{F}}_{s} \in \widetilde{\mathcal{P}}_{k+s}^{\mathbf{t}}$
j) $\nabla \bar{\mu}_{k} \cdot \widetilde{\mathbf{F}}_{r} \in \widetilde{\mathcal{P}}_{k+r}^{\mathbf{t}}$
k) $\nabla \bar{\mu}_{k} \cdot \overline{\mathbf{F}}_{s} \in \overline{\mathcal{P}}_{k+r}^{\mathbf{t}}$
1) $\left[\widetilde{\mathbf{P}}_{k}, \widetilde{\mathbf{F}}_{r}\right] \in \overline{\mathcal{Q}}_{k+r}^{\mathbf{t}}$
m) $\left[\overline{\mathbf{P}}_{k}, \widetilde{\mathbf{F}}_{r}\right] \in \widetilde{\mathcal{Q}}_{k+r}^{\mathbf{t}}$
n) $\left[\overline{\mathbf{P}}_{k}, \overline{\mathbf{F}}_{s}\right] \in \overline{\mathcal{Q}}_{k+s}^{\mathbf{t}}$
o) $\operatorname{div}\left(\widetilde{\mathbf{F}}_{r}\right) \in \widetilde{\mathcal{P}}_{r}^{\mathbf{t}}$

Proof.

- $\quad$ Item a) is trivial since $\widetilde{\mu}_{k}(-x, y) \widetilde{\mu}_{s}(-x, y)=\left(-\widetilde{\mu}_{k}(x, y)\right)\left(-\widetilde{\mu}_{s}(x, y)\right)=\widetilde{\mu}_{k}(x, y) \widetilde{\mu}_{x}(x, y)$. Therefore $\widetilde{\mu}_{k} \widetilde{\mu}_{s} \in \overline{\mathcal{P}}_{k+s}^{\mathbf{t}}$. Items b) and c) can be proved in a similar way.

- If $\widetilde{\mathbf{F}}_{r}=(\bar{P}, \widetilde{Q})^{T}$ then $\bar{P} \in \overline{\mathcal{P}}_{r+t_{1}}^{\mathbf{t}}$ and $\widetilde{Q} \in \widetilde{\mathcal{P}}_{r+t_{2}}^{\mathbf{t}}$. Therefore $\widetilde{\mu}_{k} \widetilde{\mathbf{F}}_{r}=\left(\widetilde{\mu}_{r} \bar{P}, \widetilde{\mu}_{k} \widetilde{Q}\right)^{T}$ and item d) is proved using items a) and $\mathbf{b}$ ). Items e), f) and $\mathbf{g}$ ) can be proved in an analogous manner.

- Items $\mathbf{h}$ )-k) are proved in [33] [Lemma 2.3]

- Items $\mathbf{1}$ ) and $\mathbf{m}$ ) are proved in [33] [Lemma 2.7].

- $\quad$ Item $\mathbf{n})$ can be proved using the same technique, i.e., let $\overline{\mathbf{P}}_{k}=(\widetilde{P}, \bar{Q})^{T}$ and $\overline{\mathbf{F}}_{s}=(\widetilde{F}, \bar{G})^{T}$ then $\widetilde{P} \in \widetilde{\mathcal{P}}_{k+t_{1}}^{\mathrm{t}}, \bar{Q} \in \overline{\mathcal{P}}_{k+t_{2}}^{\mathrm{t}}, \widetilde{F} \in \widetilde{\mathcal{P}}_{s+t_{1}}^{\mathrm{t}}, \bar{G} \in \overline{\mathcal{P}}_{k+t_{2}}^{\mathrm{t}}$. So, the first component of $\left[\overline{\mathbf{P}}_{k}, \overline{\mathbf{F}}_{s}\right]$, 
applying item i) is $\nabla \widetilde{P} \cdot \overline{\mathbf{F}}_{s}-\nabla \widetilde{F} \cdot \overline{\mathbf{P}}_{k} \in \overline{\mathcal{P}}_{k+s}^{\mathrm{t}}$. Analogously the second component of $\left[\overline{\mathbf{P}}_{k}, \overline{\mathbf{F}}_{s}\right]$, by item $\left.\mathbf{k}\right)$, is $\nabla \bar{Q} \cdot \overline{\mathbf{F}}_{s}-\nabla \bar{G} \cdot \overline{\mathbf{P}}_{k} \in \widetilde{\mathcal{P}}_{k+s^{\prime}}^{\mathrm{t}}$ therefore the result is obtained.

- $\quad$ Item o). If $\widetilde{\mathbf{F}}_{r} \in \widetilde{\mathcal{Q}}_{r}^{\mathbf{t}}$ then $\widetilde{\mathbf{F}}_{r}=(\bar{P}, \widetilde{Q})^{T}$ with $\bar{P} \in \overline{\mathcal{P}}_{r+t_{1}}^{\mathbf{t}}$ and $\widetilde{Q} \in \widetilde{\mathcal{P}}_{r+t_{2}}^{\mathbf{t}}$. Therefore $\frac{\partial \bar{P}}{\partial x} \in \widetilde{\mathcal{P}}_{r}^{\mathbf{t}}$ and $\frac{\partial \widetilde{Q}}{\partial y} \in \widetilde{\mathcal{P}}_{r}^{\mathrm{t}}, \operatorname{so} \operatorname{div}\left(\widetilde{\mathbf{F}}_{r}\right) \in \widetilde{\mathcal{P}}_{r}^{\mathrm{t}}$.

Proposition 1. Let be the following linear operators:

$$
\begin{aligned}
& \tilde{\ell}_{r+k}: \overline{\mathcal{P}}_{k}^{\mathbf{t}} \longrightarrow \widetilde{\mathcal{P}}_{r+k}^{\mathbf{t}} \quad \bar{\ell}_{r+k} \quad: \quad \widetilde{\mathcal{P}}_{k}^{\mathbf{t}} \longrightarrow \overline{\mathcal{P}}_{r+k}^{\mathbf{t}} \\
& \bar{\mu}_{k} \rightarrow \nabla \bar{\mu}_{k} \cdot \widetilde{\mathbf{F}}_{r} ; \quad \widetilde{\mu}_{k} \rightarrow \nabla \widetilde{\mu}_{k} \cdot \widetilde{\mathbf{F}}_{r} .
\end{aligned}
$$

Then $\operatorname{Cor}\left(\widetilde{\ell}_{r+k}\right) \oplus \operatorname{Cor}\left(\bar{\ell}_{r+k}\right)$ is a complementary subspace of $\operatorname{Range}\left(\ell_{r+k}\right)$.

Proof. As $\widetilde{\mathbf{F}}_{r}$ is $R_{x}$-reversible, by Lemma 1 items $\mathbf{h}$ ) and $\mathbf{k}$ ) the operators $\widetilde{\ell}_{r+k}$ and $\bar{\ell}_{r+k}$ are well defined. Moreover, the matrix of operator $\ell_{r+k}$ can be expressed as

\begin{tabular}{|c|c|c|}
\hline$\widetilde{\mathcal{P}}_{k}^{\mathbf{t}}$ & $\overline{\mathcal{P}}_{k}^{\mathbf{t}}$ & \\
\hline $\bar{\ell}_{r+k}\left(\widetilde{\mathcal{P}}_{k}^{\mathbf{t}}\right)$ & $\mathbf{0}$ & $\overline{\mathcal{P}}_{r+k}^{\mathbf{t}}$ \\
\hline $\mathbf{0}$ & $\widetilde{\ell}_{r+k}\left(\overline{\mathcal{P}}_{k}^{\mathbf{t}}\right)$ & $\widetilde{\mathcal{P}}_{r+k}^{\mathbf{t}}$ \\
\hline
\end{tabular}

The matrices of the reduced operators $\widetilde{\ell}_{r+k}$ and $\bar{\ell}_{r+k}$ are submatrices of the matrix of $\ell_{r+k}$ and the result is obtained.

We denote by $\mathcal{R}_{k}^{\mathbf{t}}$ a complementary subspace of $\operatorname{Ker}\left(\ell_{k-r}\right) \widetilde{\mathbf{F}}_{r}$ in $\widetilde{\mathcal{Q}}_{k}^{\mathbf{t}}$, i.e.,

$$
\widetilde{\mathcal{Q}}_{k}^{\mathrm{t}}=\operatorname{Ker}\left(\ell_{k-r}\right) \widetilde{\mathbf{F}}_{r} \bigoplus \mathcal{R}_{k}^{\mathrm{t}}
$$

Notice that, if $I \in \operatorname{Ker}\left(\ell_{r+k}\right)$, then $I$ is a polynomial first integral of $\widetilde{\mathbf{F}}_{r}$, hence $I$ must be even in the variable $x$, therefore $\operatorname{Ker}\left(\ell_{r+k}\right) \subset \overline{\mathcal{P}}_{k}^{\mathbf{t}}$ and $\operatorname{Ker}\left(\ell_{k}\right) \widetilde{\mathbf{F}}_{r} \subset \widetilde{\mathcal{Q}}_{k}^{\mathbf{t}}$. So we can define the vector space $\mathcal{R}_{k}^{\mathbf{t}}$. Moreover we can affirm that $\mathcal{Q}_{k}^{\mathbf{t}}=\mathcal{R}_{k}^{\mathrm{t}} \oplus \operatorname{Ker}\left(\ell_{k}\right) \widetilde{\mathbf{F}}_{r} \oplus \overline{\mathcal{Q}}_{k}^{\mathrm{t}}$.

We will denote $\widetilde{\Delta}_{k+|\mathbf{t}|}, \bar{\Delta}_{k+|\mathbf{t}|}$ subspaces of $\widetilde{\mathcal{P}}_{k+|\mathbf{t}|}^{\mathrm{t}}, \overline{\mathcal{P}}_{k+|\mathbf{t}|}^{\mathrm{t}}$, respectively such that $\widetilde{\mathcal{P}}_{k+|\mathbf{t}|}^{\mathrm{t}}=$ $\widetilde{\Delta}_{k+|\mathbf{t}|} \oplus h \widetilde{\mathcal{P}}_{k-r^{\prime}}^{\mathrm{t}}, \overline{\mathcal{P}}_{k+|\mathbf{t}|}^{\mathbf{t}}=\bar{\Delta}_{k+|\mathbf{t}|} \oplus h \overline{\mathcal{P}}_{k-r}^{\mathrm{t}}$. (Notice that $h \widetilde{\mathcal{P}}_{k-r}^{\mathrm{t}} \subset \widetilde{\mathcal{P}}_{k+|\mathbf{t}|}^{\mathrm{t}}$ and $h \overline{\mathcal{P}}_{k-r}^{\mathrm{t}} \subset \overline{\mathcal{P}}_{k+|\mathbf{t}|}^{\mathrm{t}}$, where $h$ is defined in (4)).

We also define the following linear operator that will be necessary in the rest of the paper:

$$
\begin{aligned}
\widetilde{\ell}_{r+k+|\mathbf{t}|}^{\mathrm{c}}: \bar{\Delta}_{k+|\mathbf{t}|} & \longrightarrow \widetilde{\Delta}_{r+k+|\mathbf{t}|} \\
g & \rightarrow \operatorname{Proy}_{\widetilde{\Delta}_{r+k+\mid \mathbf{t}}}\left(\nabla g \cdot\left(\widetilde{\mathbf{F}}_{r}-\frac{r+|\mathbf{t}|}{k+|\mathbf{t}|} \mu \mathbf{D}_{0}\right)\right) .
\end{aligned}
$$

Notice that the linear operator $\widetilde{\ell}_{i+|\mathbf{t}|}^{c}$ is well defined since we have $\mu=\frac{1}{r+|\mathbf{t}|} \operatorname{div}\left(\widetilde{\mathbf{F}}_{r}\right)(\mu$ defined in (4)) and, by Lemma 1 item o) $\mu \in \widetilde{\mathcal{P}}_{r}^{\mathbf{t}}$, then $\mu \mathbf{D}_{0} \in \widetilde{\mathcal{Q}}_{r}^{\mathbf{t}}\left(\mathbf{D}_{0}\right.$ defined in (4)). So if we consider $\widetilde{\mathbf{G}}_{r}:=\widetilde{\mathbf{F}}_{r}-\frac{r+|\mathbf{t}|}{i+|\mathbf{t}|} \mu \mathbf{D}_{0}$ then $\widetilde{\mathbf{G}}_{r} \in \widetilde{\mathcal{Q}}_{r}^{\mathrm{t}}$. Applying Lemma 1 item $\mathbf{k}$ ) we see that the operator is well defined.

\section{Orbital $\boldsymbol{R}_{x}$-Reversibility}

The following result provides a necessary and sufficient condition to have a orbitally $R_{x}$-reversible system through the orbitally $R_{x}$-reversible normal form. In addition, it allows us to construct an algorithm to compute families of orbitally $R_{x}$-reversible vector fields. This is our first main result. 
Theorem 2. Let $\mathbf{F}$ the vector field (3). If $\operatorname{Ker}\left(\widetilde{\ell}_{r+k+|\mathbf{t}|}^{\mathrm{c}}\right)=\{0\}$ for all $k \in \mathbb{N}$ then

(a) There exist $\widetilde{\mathbf{U}}=\sum_{k \geq 1} \widetilde{\mathbf{U}}_{k}, \widetilde{\mathbf{U}}_{k} \in \mathcal{R}_{k^{\prime}}^{\mathbf{t}} \widetilde{\mu}=\sum_{k \geq 1} \widetilde{\mu}_{k}, \widetilde{\mu}_{k} \in \operatorname{Cor}\left(\widetilde{\ell}_{k}\right)$ such that

$$
\widetilde{\mathbf{U}}_{* *}((1+\widetilde{\mu}) \mathbf{F})=\widetilde{\mathbf{F}}_{r}+\left(\begin{array}{c}
F\left(x^{2}, y\right) \\
x G\left(x^{2}, y\right)
\end{array}\right)+\sum_{k \geq 1}\left(\mathbf{x}_{\widetilde{g}_{r+k+\mid} \mid}+\bar{\eta}_{r+k} \mathbf{D}_{0}\right),
$$

where $\bar{\eta}_{r+k} \in \operatorname{Cor}\left(\bar{\ell}_{r+k}\right), \widetilde{g}_{r+k+|\mathbf{t}|} \in \operatorname{Cor}\left(\widetilde{\ell}_{r+k+|\mathbf{t}|}^{c}\right)$ and $\left(F\left(x^{2}, y\right), x G\left(x^{2}, y\right)\right)^{T}$ is a vector field sum of quasi-homogeneous vector fields of type $\mathbf{t}$ and degree greater than $r$.

(b) System (5) is orbitally $R_{x}$-reversible if, and only if, $\widetilde{g}_{r+k+|\mathbf{t}|}=0, \bar{\eta}_{r+k}=0$ for all $k>0$.

\subsection{Proof of Statement (a) of Theorem 2}

We can adapt an orbital normal form for generic vector field expressed in [39] [page 196], using quasi-homogeneous expansion.

Theorem 3. System $\dot{\mathbf{x}}=\widetilde{\mathbf{F}}_{r}+\sum_{k>0} \mathbf{F}_{r+k}, \mathbf{F}_{r+k} \in \mathcal{Q}_{r+k}^{\mathbf{t}}$ is formally orbital equivalent to

$$
\mathbf{y}^{\prime}=\mathbf{G}(\mathbf{y})=\widetilde{\mathbf{F}}_{r}+\sum_{k>0} \mathbf{G}_{r+k}(\mathbf{y})
$$

with $\mathbf{G}_{r+k} \in \operatorname{Cor}\left(\mathcal{L}_{r+k}\right)$, where the operator $\mathcal{L}_{r+k}$ is the homological operator under equivalence, i.e.,

$$
\begin{aligned}
\mathcal{L}_{r+k}: & \mathcal{Q}_{k}^{\mathbf{t}} \times \mathcal{P}_{k}^{\mathbf{t}} \longrightarrow \mathcal{Q}_{r+k}^{\mathbf{t}} \\
& \left(\mathbf{P}_{k}, \mu_{k}\right) \rightarrow \mathcal{L}_{r+k}\left(\mathbf{P}_{k}, \mu_{k}\right)=\left[\mathbf{P}_{k}, \widetilde{\mathbf{F}}_{r}\right]-\mu_{k} \widetilde{\mathbf{F}}_{r}
\end{aligned}
$$

In order to prove item a) of Theorem 3, we need to calculate a projection of $\operatorname{Range}\left(\mathcal{L}_{r+k}\right)$ in $\overline{\mathcal{Q}}_{r+k}^{\mathrm{t}}$ or, equivalently, to know what terms non $R_{x}$-reversible can be eliminated in the normal form. For that, we reduce the initial and the final subspaces of the homological operator. The following lemmas go in this direction.

Lemma 2. Let $k \in \mathbb{N}, \widetilde{\mathbf{F}}_{r} \in \widetilde{\mathcal{Q}}_{r}^{\mathbf{t}}, g \in \mathcal{P}_{k+|\mathbf{t}|}^{\mathbf{t}}, \eta \in \mathcal{P}_{k}^{\mathbf{t}}$ and $\lambda \in \mathcal{P}_{k-r}^{\mathbf{t}}$, then the following properties are satisfied:

(a) $\mathbf{X}_{g} \in \widetilde{\mathcal{Q}}_{k}^{\mathbf{t}}$ if, and only if, $g \in \overline{\mathcal{P}}_{k}^{\mathbf{t}}$ and $\mathbf{X}_{g} \in \overline{\mathcal{Q}}_{k}^{\mathbf{t}}$ if, and only if, $g \in \widetilde{\mathcal{P}}_{k}^{\mathbf{t}}$.

(b) $\eta \mathbf{D}_{0} \in \widetilde{\mathcal{Q}}_{k}^{\mathbf{t}}$ if, and only if, $\eta \in \widetilde{\mathcal{P}}_{k}^{\mathbf{t}}$ and $\eta \mathbf{D}_{0} \in \overline{\mathcal{Q}}_{k}^{\mathbf{t}}$ if, and only if, $\eta \in \overline{\mathcal{P}}_{k}^{\mathbf{t}}$.

(c) $\lambda \widetilde{\mathbf{F}}_{r} \in \widetilde{\mathcal{Q}}_{k}^{\mathbf{t}}$ if, and only if, $\lambda \in \overline{\mathcal{P}}_{k-r}^{\mathbf{t}}$ and $\lambda \widetilde{\mathbf{F}}_{r} \in \overline{\mathcal{Q}}_{k}^{\mathbf{t}}$ if, and only if, $\lambda \in \widetilde{\mathcal{P}}_{k-r}^{\mathbf{t}}$.

\section{Proof.}

(a) $\quad \mathbf{X}_{g}=\left(-\frac{\partial g}{\partial y}, \frac{\partial g}{\partial x}\right) \in \widetilde{\mathcal{Q}}_{k}^{\mathbf{t}}$ if, and only if, $-\frac{\partial g(-x, y)}{\partial y}=-\frac{\partial g(x, y)}{\partial y}$ and $\frac{\partial g(-x, y)}{\partial x}=-\frac{\partial g(x, y)}{\partial x}$ and this is true if, and only if, $g \in \overline{\mathcal{P}}_{k}^{\mathbf{t}}$. Analogously $\mathbf{X}_{g} \in \overline{\mathcal{Q}}_{k}^{\mathbf{t}}$ if, and only if, $-\frac{\partial g(-x, y)}{\partial y}=$ $\frac{\partial g(x, y)}{\partial y}$ and $\frac{\partial g(-x, y)}{\partial x}=\frac{\partial g(x, y)}{\partial x}$ and this is true if, and only if, $g \in \widetilde{\mathcal{P}}_{k}^{\mathbf{t}}$.

(b) $\quad \mathbf{D}_{0}=\left(t_{1} x, t_{2} y\right)^{T} \in \overline{\mathcal{Q}}_{0}^{\mathbf{t}}$, therefore by Lemma 1 items e) and $\left.\mathbf{g}\right), \eta \mathbf{D}_{0} \in \widetilde{\mathcal{Q}}_{k}^{\mathbf{t}}$ if, and only if, $\eta \in \widetilde{\mathcal{P}}_{k}^{\mathbf{t}}$. Analogously $\eta \mathbf{D}_{0} \in \overline{\mathcal{Q}}_{k}^{\mathbf{t}}$ if, and only if, $\eta \in \overline{\mathcal{P}}_{k}^{\mathbf{t}}$.

(c) $\widetilde{\mathbf{F}}_{r} \in \widetilde{\mathcal{Q}}_{r}^{\mathbf{t}}$, therefore by Lemma 1 items d) and f), $\lambda \widetilde{\mathbf{F}}_{r} \in \widetilde{\mathcal{Q}}_{k}^{\mathbf{t}}$ if, and only if, $\lambda \in \overline{\mathcal{P}}_{k-r}^{\mathbf{t}}$. Analogously $\lambda \widetilde{\mathbf{F}}_{r} \in \overline{\mathcal{Q}}_{k}^{\mathbf{t}}$ if, and only if, $\lambda \in \widetilde{\mathcal{P}}_{k-r}^{\mathbf{t}}$.

Next lemma provides the first reduction. 
Lemma 3. If $\widetilde{\mathbf{F}}_{r}$ is $R_{x}$-reversible, for all $k \in \mathbb{N}$ it is verified that $\operatorname{Proy}_{\overline{\mathcal{Q}}_{r+k}^{\mathbf{t}}}\left(\operatorname{Range}\left(\mathcal{L}_{r+k}\right)\right)=$ $\operatorname{Range}\left(\widehat{\mathcal{L}}_{r+k}\right)$ where

$$
\begin{aligned}
& \widehat{\mathcal{L}}_{r+k}: \widetilde{\mathcal{Q}}_{k}^{\mathrm{t}} \times \widetilde{\mathcal{P}}_{k}^{\mathrm{t}} \rightarrow \overline{\mathcal{Q}}_{r+k}^{\mathrm{t}} \\
& \left(\widetilde{\mathbf{U}}_{k}, \widetilde{\mu}_{k}\right) \quad-\left[\widetilde{\mathbf{F}}_{r}, \widetilde{\mathbf{U}}_{k}\right]-\widetilde{\mu}_{k} \widetilde{\mathbf{F}}_{r} .
\end{aligned}
$$

Proof. By definition $\mathcal{Q}_{k}^{\mathbf{t}} \times \mathcal{P}_{k}^{\mathbf{t}}=\left(\widetilde{\mathcal{Q}}_{k}^{\mathrm{t}} \times \widetilde{\mathcal{P}}_{k}^{\mathbf{t}}\right) \oplus\left(\overline{\mathcal{Q}}_{k}^{\mathbf{t}} \times \overline{\mathcal{P}}_{k}^{\mathbf{t}}\right)$.

With this choice we consider again the operator $\mathcal{L}_{r+k}:\left(\widetilde{\mathcal{Q}}_{k}^{\mathbf{t}} \times \widetilde{\mathcal{P}}_{k}^{\mathbf{t}}\right) \oplus\left(\overline{\mathcal{Q}}_{k}^{\mathbf{t}} \times \overline{\mathcal{P}}_{k}^{\mathbf{t}}\right) \rightarrow$ $\overline{\mathcal{Q}}_{k+r}^{\mathbf{t}} \oplus \widetilde{\mathcal{Q}}_{k+r}^{\mathrm{t}}$. As $\widetilde{\mathbf{F}}_{r} \in \widetilde{\mathcal{Q}}_{r}^{\mathbf{t}}$, applying Lemma 1 items $\left.\mathbf{d}\right), \mathbf{f}$ ), l) and $\mathbf{m}$ ), the matrix of operator $\mathcal{L}_{r+k}$ can be expressed as

\begin{tabular}{|c|c|c|}
\hline$\widetilde{\mathcal{Q}}_{k}^{\mathrm{t}} \times \widetilde{\mathcal{P}}_{k}^{\mathrm{t}}$ & $\overline{\mathcal{Q}}_{k}^{\mathrm{t}} \times \overline{\mathcal{P}}_{k}^{\mathrm{t}}$ & \\
\hline$\bullet$ & $\mathbf{0}$ & $\overline{\mathcal{Q}}_{k+r}^{\mathrm{t}}$ \\
\hline $\mathbf{0}$ & $\bullet$ & $\widetilde{\mathcal{Q}}_{k+r}^{\mathrm{t}}$ \\
\hline
\end{tabular}

where $\bullet$ means a non-null submatrix. The matrix of the reduced operator $\widehat{\mathcal{L}}_{r+k}$ is a submatrix of the matrix of $\mathcal{L}_{r+k}$ and the result is obtained.

The following lemma gives the second reduction.

Lemma 4. If $\widetilde{\mathbf{F}}_{r} \in \widetilde{\mathcal{Q}}_{r}^{\mathrm{t}}$ then for all $k \in \mathbb{N}$ it is satisfied that Range $\left(\widehat{\mathcal{L}}_{r+k}\right)=\operatorname{Range}\left(\overline{\mathcal{L}}_{r+k}\right)$ where

$$
\begin{aligned}
\overline{\mathcal{L}}_{r+k}: \mathcal{R}_{k}^{\mathbf{t}} \times \operatorname{Cor}\left(\widetilde{\ell}_{k}\right) & \rightarrow \overline{\mathcal{Q}}_{r+k}^{\mathbf{t}} \\
\left(\widetilde{\mathbf{U}}_{k}, \widetilde{v}_{k}\right) & -\left[\widetilde{\mathbf{F}}_{r}, \widetilde{\mathbf{U}}_{k}\right]-\widetilde{v}_{k} \widetilde{\mathbf{F}}_{r} .
\end{aligned}
$$

Proof. It is evident that Range $\left(\overline{\mathcal{L}}_{r+k}\right) \subset \operatorname{Range}\left(\widehat{\mathcal{L}}_{r+k}\right)$, we need to prove the other inclusion. Let $\left(\widetilde{\mathbf{U}}_{k}, \widetilde{\mu}_{k}\right) \in \widetilde{\mathcal{Q}}_{k}^{\mathbf{t}} \times \widetilde{\mathcal{P}}_{k^{\prime}}^{\mathbf{t}}$ as $\widetilde{\mathcal{Q}}_{k}^{\mathbf{t}}=\mathcal{R}_{k}^{\mathbf{t}} \oplus \operatorname{Ker}\left(\widetilde{\ell}_{k}\right) \widetilde{\mathbf{F}}_{r}$ we can affirm that there exist $\widetilde{\mathbf{V}} \in$ $\mathcal{R}_{k}^{\mathbf{t}}$ and $\bar{\lambda} \in \operatorname{Ker}\left(\widetilde{\ell}_{k}\right)$ such that $\widetilde{\mathbf{U}}_{k}=\widetilde{\mathbf{V}}_{k}+\bar{\lambda} \widetilde{\mathbf{F}}_{r}$. Moreover, as $\widetilde{\mathcal{P}}_{k}^{\mathbf{t}}=\operatorname{Range}\left(\widetilde{\ell}_{k}\right) \oplus \operatorname{Cor}\left(\widetilde{\ell}_{k}\right)$, there exist $\bar{\rho} \in \overline{\mathcal{P}}_{k}^{\mathbf{t}} \backslash \operatorname{Ker}\left(\widetilde{\ell}_{r+k}\right)$ and $\widetilde{v}_{k}^{\mathbf{t}} \in \operatorname{Cor}\left(\widetilde{\ell}_{k}\right)$ such that $\widetilde{\mu}_{k}=\nabla \bar{\rho} \cdot \widetilde{\mathbf{F}}_{r}+\widetilde{v}_{k}$. Notice that if $\bar{\rho} \in \operatorname{Ker}\left(\widetilde{\ell}_{r+k}\right)$ then $\nabla \bar{\rho} \cdot \widetilde{\mathbf{F}}_{r}=0$ and therefore $\widetilde{\mu}_{k}=0+\widetilde{v}_{k}$.

Applying the following well-known property $[\mu \mathbf{F}, \mathbf{U}]=\mu[\mathbf{F}, \mathbf{U}]+(\nabla \mu \cdot \mathbf{U}) \mathbf{F}$, we get $\left[\bar{\lambda} \widetilde{\mathbf{F}}_{r}, \widetilde{\mathbf{F}}_{r}\right]=\bar{\lambda}\left[\widetilde{\mathbf{F}}_{r}, \widetilde{\mathbf{F}}_{r}\right]+\left(\nabla \bar{\lambda} \cdot \widetilde{\mathbf{F}}_{r}\right) \mathbf{F}_{r}=\mathbf{0}$ since $\left[\widetilde{\mathbf{F}}_{r}, \widetilde{\mathbf{F}}_{r}\right]=\mathbf{0}$ and $\nabla \bar{\lambda} \cdot \widetilde{\mathbf{F}}_{r}=0$.

Applying the same property again, we get

$$
\left(\nabla \bar{\rho} \cdot \widetilde{\mathbf{F}}_{r}\right) \widetilde{\mathbf{F}}_{r}=\left[\bar{\rho} \widetilde{\mathbf{F}}_{r}, \widetilde{\mathbf{F}}_{r}\right]-\bar{\rho}\left[\widetilde{\mathbf{F}}_{r}, \widetilde{\mathbf{F}}_{r}\right]=-\left[\widetilde{\mathbf{F}}_{r}, \bar{\rho} \widetilde{\mathbf{F}}_{r}\right] .
$$

Therefore

$$
\begin{aligned}
\widehat{\mathcal{L}}_{r+k}\left(\widetilde{\mathbf{U}}_{k}, \widetilde{\mu}_{k}\right) & =-\left[\widetilde{\mathbf{F}}_{r}, \widetilde{\mathbf{V}}_{k}+\widetilde{\lambda}_{\mathbf{r}}\right]-\left(\nabla \bar{\rho} \cdot \widetilde{\mathbf{F}}_{r}+\widetilde{v}_{k}\right) \widetilde{\mathbf{F}}_{r}=-\left[\widetilde{\mathbf{F}}_{r}, \widetilde{\mathbf{V}}_{k}\right]-\left[\widetilde{\mathbf{F}}_{r}, \widetilde{\lambda}_{\mathbf{F}_{r}}\right]-\left(\nabla \bar{\rho} \cdot \widetilde{\mathbf{F}}_{r}\right) \widetilde{\mathbf{F}}_{r}-\widetilde{v}_{k} \widetilde{\mathbf{F}}_{r} \\
& =-\left[\widetilde{\mathbf{F}}_{r}, \widetilde{\mathbf{V}}_{k}\right]+\left[\bar{\lambda} \widetilde{\mathbf{F}}_{r}, \widetilde{\mathbf{F}}_{r}\right]+\left[\widetilde{\mathbf{F}}_{r}, \widetilde{\mathbf{F}}_{r}\right]-\widetilde{v}_{k} \widetilde{\mathbf{F}}_{r}=-\left[\widetilde{\mathbf{F}}_{r}, \widetilde{\mathbf{V}}_{k}-\bar{\rho} \widetilde{\mathbf{F}}_{r}\right]-\widetilde{v}_{k} \widetilde{\mathbf{F}}_{r} \\
& =\overline{\mathcal{L}}_{r+k}\left(\widetilde{\mathbf{W}}_{k}, \widetilde{v}_{k}\right) .
\end{aligned}
$$

where $\widetilde{\mathbf{W}}_{k}=\widetilde{\mathbf{V}}_{k}-\bar{\rho} \widetilde{\mathbf{F}}_{r} \in \mathcal{R}_{k^{\prime}}^{\mathrm{t}}$ since $\bar{\rho} \notin \operatorname{Ker}\left(\widetilde{\ell}_{r+k}\right)$, and the result is obtained. 
In order to calculate a complementary subspace to Range $\left(\overline{\mathcal{L}}_{r+k}\right)$, we need to define the following subspaces (see Properties of Lemma 2)

$$
\begin{array}{ll}
\widetilde{\mathcal{C}}_{k}=\left\{\mathbf{x}_{g_{k+\mid} \mid \mathbf{t}}: g_{k+|\mathbf{t}|} \in \bar{\Delta}_{k+|\mathbf{t}|}\right\}, & \overline{\mathcal{C}}_{k}=\left\{\mathbf{x}_{\left.g_{k+\mid \mathbf{t}}: g_{k+|\mathbf{t}|} \in \widetilde{\Delta}_{k+|\mathbf{t}|}\right\},}\right. \\
\widetilde{\mathcal{D}}_{k}=\left\{\eta_{k} \mathbf{D}_{0}: \eta_{k} \in \widetilde{\mathcal{P}}_{k}^{\mathbf{t}}\right\}, & \overline{\mathcal{D}}_{k}=\left\{\eta_{k} \mathbf{D}_{0}: \eta_{k} \in \overline{\mathcal{P}}_{k}^{\mathbf{t}}\right\}, \\
\widetilde{\mathcal{F}}_{k}=\left\{\lambda_{k-r} \widetilde{\mathbf{F}}_{r}: \lambda_{k-r} \in \overline{\mathcal{P}}_{k-r}^{\mathbf{t}} \backslash \operatorname{Ker}\left(\ell_{k}\right) \widetilde{\mathbf{F}}_{r}\right\}, & \overline{\mathcal{F}}_{k}=\left\{\lambda_{k-r} \widetilde{\mathbf{F}}_{r}: \lambda_{k-r} \in \widetilde{\mathcal{P}}_{k-r}^{\mathbf{t}}\right\} .
\end{array}
$$

Notice that $\mathcal{P}_{k-r}^{\mathbf{t}}=\{0\}$ if $k<r$ and $\mathcal{P}_{0}^{\mathbf{t}}=\operatorname{span}\{1\}$.

Next result is an adaptation to our case of [40] [Lemma 3.6].

Lemma 5. If $\mathbf{D}_{0} \wedge \widetilde{\mathbf{F}}_{r} \neq 0$, then $\mathcal{R}_{k}^{\mathbf{t}}=\widetilde{\mathcal{C}}_{k}^{\mathbf{t}} \oplus \widetilde{\mathcal{D}}_{k}^{\mathbf{t}} \oplus \widetilde{\mathcal{F}}_{k}^{\mathbf{t}}$ and $\overline{\mathcal{Q}}_{k}^{\mathbf{t}}=\overline{\mathcal{C}}_{k}^{\mathbf{t}} \oplus \overline{\mathcal{D}}_{k}^{\mathbf{t}} \oplus \overline{\mathcal{F}}_{k}^{\mathbf{t}}$, for all $k \in \mathbb{N}$. Moreover if $\widetilde{\mathbf{P}}_{k} \in \mathcal{R}_{k}^{\mathbf{t}}, \overline{\mathbf{P}}_{k} \in \overline{\mathcal{Q}}_{k}^{\mathbf{t}}$, there exist $\bar{g} \in \bar{\Delta}_{k+|\mathbf{t}|}, \widetilde{\eta} \in \mathcal{P}_{k}^{\mathbf{t}}$ and $\bar{\lambda} \in \overline{\mathcal{P}}_{k-r}^{\mathbf{t}}$ such that

$$
\begin{aligned}
& \widetilde{\mathbf{P}}_{k}=\mathbf{X}_{\bar{g}}+\widetilde{\eta} \mathbf{D}_{0}+\bar{\lambda} \mathbf{F}_{r}, \\
& \overline{\mathbf{P}}_{k}=\mathbf{X}_{\widetilde{g}}+\bar{\eta} \mathbf{D}_{0}+\widetilde{\lambda} \mathbf{F}_{r},
\end{aligned}
$$

where

$$
\begin{gathered}
\bar{g}=\frac{\operatorname{Proy}_{\bar{\Delta}_{k+|| t \mid}} \mathbf{D}_{0} \wedge \widetilde{\mathbf{P}}_{k}}{k+|\mathbf{t}|}, \bar{\lambda}=\frac{\operatorname{Proy}_{h \bar{P}_{k-r}^{t}} \mathbf{D}_{0} \wedge \widetilde{\mathbf{P}}_{k}}{(r+|\mathbf{t}|) h}, \bar{\eta}=\frac{\operatorname{div}\left(\widetilde{\mathbf{P}}_{k}\right)-\nabla \bar{\lambda} \cdot \mathbf{F}_{r}-\bar{\lambda} \operatorname{div}\left(\mathbf{F}_{r}\right)}{r+|\mathbf{t}|}, \\
\widetilde{g}=\frac{\operatorname{Proy}_{\widetilde{\Delta}_{k+|| t \mid}} \mathbf{D}_{0} \wedge \overline{\mathbf{P}}_{k}}{k+|\mathbf{t}|}, \tilde{\lambda}=\frac{\operatorname{Proy}_{h \widetilde{k}_{k-r}^{t}} \mathbf{D}_{0} \wedge \overline{\mathbf{P}}_{k}}{(r+\mid \mathbf{t}) h}, \bar{\eta}=\frac{\operatorname{div}\left(\overline{\mathbf{P}}_{k}\right)-\nabla \tilde{\lambda} \cdot \mathbf{F}_{r}-\widetilde{\lambda} \operatorname{div}\left(\mathbf{F}_{r}\right)}{r+|\mathbf{t}|} .
\end{gathered}
$$

Next result uses the above decompositions for calculating a complementary subspace of Range $\left(\overline{\mathcal{L}}_{r+k}\right)$ and, therefore, for computing a normal form for detecting the orbital reversibility of a vector field.

Proposition 2. If $\widetilde{\mathbf{F}}_{r} \in \widetilde{\mathcal{Q}}_{r}^{\mathbf{t}}$ and $\operatorname{Ker}\left(\widetilde{\ell}_{r+k+|\mathbf{t}|}^{\mathrm{c}}\right)=\{0\}$ for all $k \in \mathbb{N}$, then a complementary subspace to range of $\overline{\mathcal{L}}_{r+k}$ is $\operatorname{Cor}\left(\overline{\mathcal{L}}_{r+k}\right)=\mathbf{X}_{\widetilde{g}}+\bar{\eta} \mathbf{D}_{0}$, where $\widetilde{g} \in \operatorname{Cor}\left(\widetilde{\ell}_{r+k+|\mathbf{t}|}^{c}\right)$ and $\bar{\eta} \in$ $\operatorname{Cor}\left(\bar{\ell}_{r+k}\right)$. Moreover $\operatorname{Ker}\left(\overline{\mathcal{L}}_{r+k}\right)=\{\mathbf{0}\}$

Proof. By Lemma $5 \mathcal{R}_{k}^{\mathbf{t}}=\widetilde{\mathcal{C}}_{k}^{\mathrm{t}} \oplus \widetilde{\mathcal{D}}_{k}^{\mathrm{t}} \oplus \widetilde{\mathcal{F}}_{k^{\prime}}^{\mathrm{t}} \overline{\mathcal{Q}}_{r+k}^{\mathbf{t}}=\overline{\mathcal{C}}_{r+k}^{\mathbf{t}} \oplus \overline{\mathcal{D}}_{r+k}^{\mathbf{t}} \oplus \overline{\mathcal{F}}_{r+k}^{\mathbf{t}}$. On the other hand $\overline{\mathcal{P}}_{r+k}^{\mathrm{t}}=\operatorname{Range}\left(\widetilde{\ell}_{k}\right) \oplus \operatorname{Cor}\left(\widetilde{\ell}_{k}\right)$ then $\overline{\mathcal{F}}_{r+k}^{\mathrm{t}}=\operatorname{Range}\left(\widetilde{\ell}_{k}\right) \widetilde{\mathbf{F}}_{r} \oplus \operatorname{Cor}\left(\widetilde{\ell}_{k}\right) \widetilde{\mathbf{F}}_{r}$. Moreover, the following properties, that are demonstrated below, are verified

(a) $-\left[\widetilde{\mathbf{F}}_{r}, \bar{\lambda}_{k-r} \widetilde{\mathbf{F}}_{r}\right]=\bar{\ell}_{r+k}\left(\bar{\lambda}_{k-r}\right) \in \overline{\mathcal{F}}_{r+k}^{\mathbf{t}}$ if $\bar{\lambda}_{k-r} \in \overline{\mathcal{P}}_{k-r}^{\mathbf{t}}$,

(b) $\quad-\left[\widetilde{\mathbf{F}}_{r}, \bar{\eta}_{k} \mathbf{D}_{0}\right]=\bar{\ell}_{r+k}\left(\widetilde{\eta}_{k}\right) \mathbf{D}_{0}-r \widetilde{\eta}_{k} \widetilde{\mathbf{F}}_{r} \in \overline{\mathcal{D}}_{r+k}^{\mathbf{t}} \oplus \overline{\mathcal{F}}_{r+k}^{\mathbf{t}}$ if $\widetilde{\eta}_{k} \in \widetilde{\mathcal{P}}_{k^{\prime}}^{\mathbf{t}}$

(c) $\operatorname{Proy}_{\overline{\mathcal{C}}_{r+k}}\left(-\left[\widetilde{\mathbf{F}}_{r}, \mathbf{X}_{\bar{g}}\right]\right)=\mathbf{X}_{\widetilde{\ell}_{r+k+|\mathfrak{t}|}^{c}}$ if $)$ if $\bar{g} \in \bar{\Delta}_{k+|\mathbf{t}|}$.

Items (a) and (b), are consequence of the following properties $[\mu \mathbf{F}, \mathbf{G}]=(\nabla \mu \cdot \mathbf{G}) \mathbf{F}+$ $\mu[\mathbf{F}, \mathbf{G}]$ and $\left[\mathbf{F}_{k}, \mathbf{D}_{0}\right]=k \mathbf{F}_{k}$, respectively. From the properties of the Lie bracket and the previous properties we deduce 


$$
\begin{aligned}
{\left[\widetilde{\mathbf{F}}_{r}, \mathbf{X}_{\bar{g}}\right] } & =\left[\mathbf{X}_{h}, \mathbf{X}_{\bar{g}}\right]+\left[\mu \mathbf{D}_{0}, \mathbf{X}_{\bar{g}}\right]=\mathbf{X}_{-\nabla \bar{g} \cdot \mathbf{X}_{h}}+\left(\nabla \mu \cdot \mathbf{X}_{\bar{g}}\right) \mathbf{D}_{0}+\mu\left[\mathbf{D}_{0}, \mathbf{X}_{\bar{g}}\right] \\
& =\mathbf{X}_{-\nabla \bar{g} \cdot \mathbf{X}_{h}}+\left(\nabla \mu \cdot \mathbf{X}_{\bar{g}}\right) \mathbf{D}_{0}-k \mu \mathbf{X}_{\bar{g}} \\
& =\mathbf{X}_{-\nabla \bar{g} \cdot \mathbf{X}_{h}}+\left(\nabla \mu \cdot \mathbf{X}_{\bar{g}}\right) \mathbf{D}_{0}-\frac{k(k+\mid \mathbf{t}))}{r+k+|\mathbf{t}|} \mathbf{X}_{\mu \bar{g}}-\frac{k}{r+k+|\mathbf{t}|}\left(\nabla \mu \cdot \mathbf{X}_{\bar{g}}\right) \mathbf{D}_{0} \\
& =-\mathbf{X}_{\nabla \bar{g} \cdot \mathbf{X}_{h}+\frac{k(k+|\mathbf{t}|)}{r+k+|\mathbf{t}|} \mu \bar{g}}+\frac{r+|\mathbf{t}|}{r+k+|\mathbf{t}|}\left(\nabla \mu \cdot \mathbf{X}_{\bar{g}}\right) \mathbf{D}_{0} \\
& =-\mathbf{X}_{\nabla \bar{g} \cdot\left(\mathbf{F}_{r}-\frac{r+|\mathbf{t}|}{r+k+\mid \mathbf{t}} \mu \mathbf{D}_{0}\right)}+\frac{r+|\mathbf{t}|}{r+k+|\mathbf{t}|}\left(\nabla \mu \cdot \mathbf{X}_{\bar{g}}\right) \mathbf{D}_{0}
\end{aligned}
$$

which implies item (c).

From (a)-(c) and using Lemma 1 we obtain the following scheme for $\overline{\mathcal{L}}_{r+k}$ :

\begin{tabular}{|c|c|c|c|c|}
\hline$\widetilde{\mathcal{C}}_{k}^{\mathbf{t}}$ & $\widetilde{\mathcal{D}}_{k}^{\mathbf{t}}$ & $\widetilde{\mathcal{F}}_{k}^{\mathrm{t}}$ & $\operatorname{Cor}\left(\widetilde{\ell}_{k}\right)$ & \\
\hline $\mathbf{X}_{\left.\left(\widetilde{\ell}_{r+k+\mid \mathbf{t}}^{\mathrm{c}} \bar{g}^{\mathrm{g}}\right)\right)}$ & 0 & 0 & 0 & $\overline{\mathcal{C}}_{r+k}^{\mathbf{t}}$ \\
\hline$-\left[\widetilde{\mathbf{F}}_{r}, \mathbf{X}_{\bar{g}}\right]^{\mathrm{d}}$ & $\bar{\ell}_{r+k}\left(\widetilde{\eta}_{k}\right) \mathbf{D}_{0}$ & 0 & 0 & $\overline{\mathcal{D}}_{r+k}^{\mathbf{t}}$ \\
\hline \multirow{2}{*}{$-\left[\widetilde{\mathbf{F}}_{r}, \mathbf{X}_{\bar{g}}\right]^{\mathrm{f}}$} & $-r \widetilde{\eta}_{k} \widetilde{\mathbf{F}}_{r}$ & $\widetilde{\ell}_{k}\left(\bar{\lambda}_{k-r}\right) \widetilde{\mathbf{F}}_{r}$ & 0 & $\operatorname{Range}\left(\widetilde{\ell}_{k}\right) \widetilde{\mathbf{F}}_{r}$ \\
\cline { 3 - 4 } & & 0 & $-\widetilde{v}_{k} \widetilde{\mathbf{F}}_{r}$ & $\operatorname{Cor}\left(\widetilde{\ell}_{k}\right) \widetilde{\mathbf{F}}_{r}$ \\
\hline
\end{tabular}

where $\left[\widetilde{\mathbf{F}}_{r}, \mathbf{X}_{\bar{g}}\right]^{\mathrm{d}}=\operatorname{Proy}_{\overline{\mathcal{D}}_{r+k}}\left(\left[\widetilde{\mathbf{F}}_{r}, \mathbf{X}_{\bar{g}}\right]\right)$ and $\left[\widetilde{\mathbf{F}}_{r}, \mathbf{X}_{\bar{g}}\right]^{\mathrm{f}}=\operatorname{Proy}_{\overline{\mathcal{F}}_{r+k}}\left(\left[\widetilde{\mathbf{F}}_{r}, \mathbf{X}_{\bar{g}}\right]\right)$. From hypothesis $\operatorname{Ker}\left(\widetilde{\ell}_{r+k+|\mathbf{t}|}^{c}\right)=\{0\}$, we can deduce that the upper left block of the above matrix has maximum range. Therefore $\operatorname{Cor}\left(\overline{\mathcal{L}}_{r+k}\right)$ follows from the structure of the above matrix.

Moreover all others blocks of the above matrix have maximum range. The first block by hypothesis, the second block since, if there exists $\widetilde{\eta}_{k} \in \widetilde{\mathcal{P}}_{k}^{\mathrm{t}}$ such that $\bar{\ell}_{r+k}\left(\widetilde{\eta}_{k}\right)=0$ then $\ell_{r+k}\left(\widetilde{\eta}_{k}\right)=0$, then $\widetilde{\eta}_{k}$ is a polynomial first integral of $\widetilde{\mathbf{F}}_{r}$, hence $\widetilde{\eta}_{k}$ must be even in the variable $x$, therefore $\widetilde{\eta} \in \widetilde{\mathcal{P}}_{k}^{\mathrm{t}} \cap \overline{\mathcal{P}}_{k}^{\mathrm{t}}=\{0\}$ and it is a contradiction. The third block has maximum range, otherwise there exists $\bar{\lambda}_{k-r} \in \overline{\mathcal{P}}_{k-r}^{\mathrm{t}}$ such that $\widetilde{\ell}_{k}\left(\bar{\lambda}_{k-r}\right)=0$, i.e., $\ell_{k}\left(\bar{\lambda}_{k-r}\right)=0$ but it is impossible since $\widetilde{\mathcal{F}}_{k}=\operatorname{Proy}_{\mathcal{R}_{k}^{\mathrm{t}}}\left(\mathcal{F}_{k}\right)$ and $\widetilde{\mathcal{Q}}_{k}^{\mathrm{t}}=\mathcal{R}_{k}^{\mathrm{t}} \oplus \operatorname{Ker}\left(\ell_{k}\right) \widetilde{\mathbf{F}}_{r}$. It is evident that the last block has maximum range. Therefore $\operatorname{Ker}\left(\overline{\mathcal{L}}_{r+k}\right)=\{\mathbf{0}\}$.

Proof. [Statement (a) of Theorem 2] By Theorem 3 a formally orbital normal form of system $\dot{\mathbf{x}}=\mathbf{F}(\mathbf{x})$ is $\dot{\mathbf{x}}=\mathbf{G}(\mathbf{x}):=\mathbf{F}_{r}+\sum_{k>0} \mathbf{G}_{r+k}$, with $\mathbf{G}_{r+k} \in \operatorname{Cor}\left(\mathcal{L}_{r+k}\right)$ (complementary space to the range of $\mathcal{L}_{r+k}$ ). From Lemma 3 it is possible to choose a complementary subspace such that $\operatorname{Proy}_{\overline{\mathcal{Q}}_{r+k}^{t}}\left(\operatorname{Cor}\left(\mathcal{L}_{r+k}\right)\right)=\operatorname{Cor}\left(\widehat{\mathcal{L}}_{r+k}\right)$, see the matrix of the operator $\mathcal{L}_{r+k}$ described in the demonstration of the cited lemma. By Lemma $4 \operatorname{Cor}\left(\widehat{\mathcal{L}}_{r+k}\right)=\operatorname{Cor}\left(\overline{\mathcal{L}}_{r+k}\right)$. To finish the proof it is enough to apply the Proposition 2.

\subsection{Proof of Statement (b) of Theorem 2}

We begin this section by demonstrating some results (technical lemmas) regarding the theory of normal forms that will be necessary.

The following result states that the order in which we apply the spatial and temporal generators does not matter.

Lemma 6. Let us consider $\mu_{k} \in \mathcal{P}_{k}^{\mathbf{t}}(k \in \mathbb{N})$ and $\mathbf{U} \in \bigoplus_{j \geq 1} \mathcal{Q}_{j}^{\mathbf{t}}$. Then, there exists $\lambda=\sum_{j \geq k} \lambda_{j} \in$ $\bigoplus_{j \geq k} \mathcal{P}_{j}^{\mathbf{t}}$, such that

$$
\left(1+\mu_{k}\right)\left(\mathbf{U}_{* *} \mathbf{F}\right)=\mathbf{U}_{* *}((1+\lambda) \mathbf{F}),
$$

where the lowest-degree quasi-homogeneous term of $\lambda$ is $\lambda_{k}=\mu_{k}$. 
Proof. Let us denote the successive Lie derivatives along the vector field $\mathbf{U}$ of the scalar function $\mu_{k}$ by $\mu_{k}^{[j]}$, i.e.,

$$
\mu_{k}^{[0]}=\mu_{k}, \text { and } \mu_{k}^{[j]}=\nabla \mu_{k}^{[j-1]} \cdot \mathbf{U}, \text { for } j \geq 1 .
$$

Using induction, we can prove that

$$
\mu_{k} T_{\mathbf{U}}^{(l)}(\mathbf{F})=\sum_{j=0}^{l}\left(\begin{array}{l}
l \\
j
\end{array}\right)(-1)^{j} T_{\mathbf{U}}^{(l-j)}\left(\mu_{k}^{[j]} \mathbf{F}\right), \text { for all } l \geq 0 .
$$

Then,

$$
\begin{aligned}
\left(1+\mu_{k}\right)\left(\mathbf{U}_{* *} \mathbf{F}\right) & =\sum_{l=0}^{\infty} \frac{1}{l !} T_{\mathbf{U}}^{(l)}(\mathbf{F})+\sum_{l=0}^{\infty} \frac{1}{l !} \sum_{j=0}^{l}\left(\begin{array}{l}
l \\
j
\end{array}\right)(-1)^{j} T_{\mathbf{U}}^{(l-j)}\left(\mu_{k}^{[j]} \mathbf{F}\right) \\
& =\sum_{l=0}^{\infty} \frac{1}{l !} T_{\mathbf{U}}^{(l)}(\mathbf{F})+\sum_{l=0}^{\infty} \sum_{j=0}^{l} \frac{1}{j ! l !}(-1)^{j} T_{\mathbf{U}}^{(l)}\left(\mu_{k}^{[j]} \mathbf{F}\right) \\
& =\sum_{l=0}^{\infty} \frac{1}{l !} T_{\mathbf{U}}^{(l)}\left(\left(1+\sum_{j=0}^{l} \frac{1}{j !}(-1)^{j} \mu_{k}^{[j]}\right) \mathbf{F}\right)=\mathbf{U}_{* *}((1+\lambda) \mathbf{F}),
\end{aligned}
$$

as claimed, where $\lambda:=\sum_{j=0}^{l} \frac{1}{j !}(-1)^{j} \mu_{k}^{[j]}$. Moreover, it is easy to show that $\lambda_{k}=\mu_{k}$.

The following results are technical lemmas that will be used later.

Lemma 7. Let us consider a couple of generators: $\mathbf{U}=\sum_{j \geq 1} \mathbf{U}_{j} \in \bigoplus_{j \geq 1} \mathcal{Q}_{j}^{\mathbf{t}}$ and $\mathbf{V}=\sum_{j \geq k} \mathbf{V}_{j} \in$ $\bigoplus_{j \geq k} \mathcal{Q}_{j}^{\mathbf{t}}(k \in \mathbb{N})$, corresponding to near-identity transformations $\Phi$ and $\Psi$, respectively. If $\mathbf{W}=$ $\sum_{j \geq 1} \mathbf{W}_{j} \in \bigoplus_{j \geq 1} \mathcal{Q}_{j}^{\mathbf{t}}$ is a generator of $\Psi \circ \Phi$, then $\mathbf{U}$ and $\mathbf{W}$ agree up to quasi-homogeneous degree $k-1$ (i.e., $\mathcal{J}^{k-1}(\mathbf{U})=\mathcal{J}^{k-1}(\mathbf{W})$ ), and

$$
\mathbf{W}_{k}=\mathbf{U}_{k}+\mathbf{V}_{k} .
$$

Proof. The proof can be found in [33] [Lemma 2.8].

Lemma 8. Let us consider $\rho_{m} \in \mathcal{P}_{m}^{\mathbf{t}}(m \in \mathbb{N})$ and $\mu \in \bigoplus_{j \geq 1} \mathcal{P}_{j}^{\mathbf{t}}$. Then, there exists $\eta=$ $\sum_{j \geq 1} \eta_{j} \in \bigoplus_{j \geq 1} \mathcal{P}_{j}^{\mathbf{t}}$, such that:

$$
\left(\rho_{m} \mathbf{F}\right)_{* *}((1+\mu) \mathbf{F})=(1+\eta) \mathbf{F},
$$

where $\eta$ and $\mu$ agree up to quasi-homogeneous degree $m+r-1\left(\right.$ i.e., $\mathcal{J}^{m+r-1}(\eta)=\mathcal{J}^{m+r-1}(\mu)$ ), and $\eta_{m+r}=\mu_{m+r}-\nabla \rho_{m} \cdot \widetilde{\mathbf{F}}_{r}$.

Proof. Firstly, we will show using induction that, for each $l \in \mathbb{N}$, there exists $\eta^{(l)} \in$ $\bigoplus_{j \geq l(m+r)} \mathcal{P}_{j}^{\mathrm{t}}$ satisfying:

$$
T_{\rho_{m} \mathbf{F}}^{(l)}((1+\mu) \mathbf{F})=\eta^{(l)} \mathbf{F},
$$

where $\eta_{m+r}^{(1)}=-\nabla \rho_{m} \cdot \widetilde{\mathbf{F}}_{r}$.

Namely, for $l=1$, applying the following well-known property $[\mu \mathbf{F}, \mathbf{G}]=(\nabla \mu \cdot \mathbf{G}) \mathbf{F}+$ $\mu[\mathbf{F}, \mathbf{G}]$, we deduce:

$$
T_{\rho_{m} \mathbf{F}}((1+\mu) \mathbf{F})=\left[(1+\mu) \mathbf{F}, \rho_{m} \mathbf{F}\right]=\left(\rho_{m}(\nabla \mu \cdot \mathbf{F})-(1+\mu)\left(\nabla \rho_{m} \cdot \mathbf{F}\right)\right) \mathbf{F},
$$

and it is enough to take $\eta^{(1)}:=\rho_{m}(\nabla \mu \cdot \mathbf{F})-(1+\mu)\left(\nabla \rho_{m} \cdot \mathbf{F}\right)$. Equating quasi-homogeneous terms, we get $\eta_{i}^{(1)}=0$ for $i=1, \cdots, m+r-1$, and $\eta_{m+r}^{(1)}=-\nabla \rho_{m} \cdot \widetilde{\mathbf{F}}_{r}$. 
Next, we assume that (6) holds for $l-1$. Applying again $[\mu \mathbf{F}, \mathbf{G}]=\mu[\mathbf{F}, \mathbf{G}]+(\nabla \mu$. G) F, we obtain:

$$
T_{\rho_{m} \mathbf{F}}^{(l)}((1+\mu) \mathbf{F})=\left[T_{\rho_{m} \mathbf{F}}^{(l-1)}((1+\mu) \mathbf{F}), \rho_{m} \mathbf{F}\right]=\left[\eta^{(l-1)} \mathbf{F}, \rho_{m} \mathbf{F}\right]=\eta^{(l)} \mathbf{F},
$$

where we have denoted $\eta^{(l)}:=\rho_{m}\left(\nabla \eta^{(l-1)} \cdot \mathbf{F}\right)-\eta^{(l-1)}\left(\nabla \rho_{m} \cdot \mathbf{F}\right)$. As $\eta^{(l-1)} \in$ $\bigoplus_{j \geq(l-1)(m+r)} \mathcal{P}_{j}^{\mathbf{t}}$, it is easy to show that $\eta^{(l)} \in \bigoplus_{j \geq l(m+r)} \mathcal{P}_{j}^{\mathbf{t}}$. This proves the equality (6).

From this equality, we obtain:

$$
\begin{aligned}
\left(\rho_{m} \mathbf{F}\right)_{* *}((1+\mu) \mathbf{F}) & =\sum_{l=0}^{\infty} \frac{1}{l !} T_{\rho_{m}}^{(l)} \mathbf{F}((1+\mu) \mathbf{F})=(1+\mu) \mathbf{F}+\sum_{l=0}^{\infty} \frac{1}{l !} \eta^{(l)} \mathbf{F} \\
& =\left(1+\mu+\sum_{l=1}^{\infty} \frac{1}{l !} \eta^{(l)}\right) \mathbf{F}=(1+\eta) \mathbf{F}
\end{aligned}
$$

where we have introduced $\eta:=\mu+\sum_{l \geq 1} \frac{1}{l !} \eta^{(l)}$.

Proposition 3. Let $N \in \mathbb{N}$ and $\mathbf{F}=\widetilde{\mathbf{F}}_{r}+\cdots$, where $\widetilde{\mathbf{F}}_{r}$ is $R_{x}$-reversible and $\mathbf{U} \in \bigoplus_{j \geq 1} \mathcal{Q}_{j}^{\mathbf{t}}, \mu \in$ $\bigoplus_{j \geq 1} \mathcal{P}_{j}^{\mathbf{t}}$ such that $\mathcal{J}^{r+N-1}\left(\mathbf{U}_{* *}((1+\mu) \mathbf{F})\right)$ is $R_{x}$-reversible. Then, there exist $\mathbf{W}=\sum_{j \geq 1} \mathbf{W}_{j}$, $\mathbf{W}_{j} \in \mathcal{Q}_{j}^{\mathbf{t}}, \mathcal{J}^{N}(\mathbf{W}) \in \bigoplus_{j=1}^{N} \mathcal{R}_{j}^{\mathbf{t}}$ and $\delta=\sum_{j \geq 1} \delta_{j}, \delta_{j} \in \mathcal{P}_{j}^{\mathbf{t}}, \mathcal{J}^{N}(\delta)=\bigoplus_{j=1}^{N} \operatorname{Cor}\left(\widetilde{\ell}_{j}\right)$ satisfying

$$
\mathcal{J}^{r+N}\left(\overline{\mathbf{W}_{* *}((1+\delta) \mathbf{F})}\right)=\mathcal{J}^{r+N}\left(\overline{\mathbf{U}_{* *}((1+\mu) \mathbf{F})}\right),
$$

where $\mathcal{J}^{M}(\mathbf{W})$ denotes the $M$-jet of $\mathbf{W}$, i.e., $\mathcal{J}^{M}(\mathbf{W}):=\mathbf{W}_{1}+\cdots+\mathbf{W}_{M}$ being $\mathbf{W}=\mathbf{W}_{1}+$ $\cdots+\mathbf{W}_{M}+\cdots \in \bigoplus_{j \geq 1} \mathcal{Q}_{j}^{\mathbf{t}}$ and $\mathcal{J}^{M}(\delta)$ denotes the $M$-jet of $\delta$, i.e., $\mathcal{J}^{M}(\delta):=\delta_{1}+\cdots+\delta_{M}$ being $\delta=\delta_{1}+\cdots+\delta_{M}+\cdots$.

Proof. Let us denote

$$
\kappa=\min \left\{k \in \mathbb{N}: \operatorname{Proy}_{\overline{\mathcal{Q}}_{k}^{\mathrm{t}} \oplus \operatorname{Ker}\left(\ell_{k}\right) \widetilde{\mathbf{F}}_{r}}\left(\mathbf{U}_{k}\right) \neq \mathbf{0}, \quad \text { or } \operatorname{Proy}_{\overline{\mathcal{P}}_{k}^{\mathrm{t}} \oplus \operatorname{Range}\left(\widetilde{\ell}_{k}\right)}\left(\mu_{k}\right) \neq 0\right\} .
$$

We assume $\kappa<N$ (otherwise, as $\mathcal{Q}_{j}^{\mathbf{t}}=\mathcal{R}_{j}^{\mathbf{t}} \oplus \operatorname{Ker}\left(\ell_{j}\right) \widetilde{\mathbf{F}}_{r} \oplus \overline{\mathcal{Q}}_{j}^{\mathbf{t}}$ and $\mathcal{P}_{j}^{\mathbf{t}}=\operatorname{Cor}\left(\widetilde{\ell}_{j}\right)$ $\left.\oplus \operatorname{Range}\left(\tilde{\ell}_{j}\right) \oplus \overline{\mathcal{P}}_{j}^{\mathbf{t}}\right)$, taking $\mathbf{W}=\mathbf{U}, \delta=\mu$, we complete the proof.

From the definition of $\kappa$, we get that $\mathbf{U}_{j} \in \mathcal{R}_{j}^{\mathbf{t}}$ and $\mu_{j} \in \operatorname{Cor}\left(\widetilde{\ell}_{j}\right)$ for $j=1, \ldots, \kappa-1$. Our goal is to show that the result is true for the $\kappa$-degree quasi-homogeneous terms and then, repeating the procedure, we get the result.

We can write $\mathbf{U}_{\kappa}=\overline{\mathbf{U}}_{\kappa}+\widetilde{\mathbf{U}}_{\kappa}$, where $\overline{\mathbf{U}}_{\kappa} \in \overline{\mathcal{Q}}_{\kappa}^{\mathrm{t}}$ and $\widetilde{\mathbf{U}}_{\kappa} \in \widetilde{\mathcal{Q}}_{\kappa}^{\mathrm{t}}$. Also, we can split $\mu_{\kappa}=\bar{\mu}_{\kappa}+\widetilde{\mu}_{\kappa}$, with $\bar{\mu}_{\kappa} \in \overline{\mathcal{P}}_{\kappa}^{\mathrm{t}}$ and $\widetilde{\mu}_{\kappa} \in \widetilde{\mathcal{P}}_{\kappa}^{\mathrm{t}}$.

Let us denote by $\mathbf{V}$ a generator of $\Psi \circ \Phi$, where $\Phi$ and $\Psi$ are the transformations generated by $\mathbf{U}$ and $-\overline{\mathbf{U}}_{\kappa}$, respectively. From Lemma 7, we have $\mathcal{J}^{\kappa-1}(\mathbf{V})=\mathcal{J}^{\kappa-1}(\mathbf{U})$ and

$$
\mathbf{V}_{\kappa}=\mathbf{U}_{\kappa}-\overline{\mathbf{U}}_{\kappa}=\widetilde{\mathbf{U}}_{\kappa} \in \widetilde{\mathcal{Q}}_{\kappa}^{\mathbf{t}} .
$$

Therefore $\mathcal{J}^{\kappa}(\mathbf{V}) \in \bigoplus_{j=1}^{\kappa} \mathcal{R}_{j}^{\mathrm{t}}$.

On the other hand, by Lemma 1 item $\mathbf{n}$ ) it is verified that $\left[\overline{\mathbf{F}}_{k}, \overline{\mathbf{U}}_{j}\right] \in \overline{\mathcal{Q}}_{k+j}^{\mathbf{t}}$. So we get:

$$
\begin{aligned}
\overline{\mathbf{V}_{* *}((1+\mu) \mathbf{F})} & =\overline{(\Psi \circ \Phi)_{*}((1+\mu) \mathbf{F})}=\overline{\Psi_{*}\left(\mathbf{U}_{* *}((1+\mu) \mathbf{F})\right)} \\
& =\overline{-\overline{\mathbf{U}}_{\kappa * *}\left(\mathbf{U}_{* *}((1+\mu) \mathbf{F})\right)}=\left(-\overline{\mathbf{U}}_{\kappa}\right)_{* *}\left(\overline{\mathbf{U}_{* *}((1+\mu) \mathbf{F})}\right) .
\end{aligned}
$$


we get

As $\mathcal{J}^{r+m-1}\left(\overline{\mathbf{U}_{* *}((1+\mu) \mathbf{F})}\right)=\mathbf{0}$ since $\mathcal{J}^{r+m-1}\left(\mathbf{U}_{* *}((1+\mu) \mathbf{F})\right)$ is $R_{x}$-reversible,

$$
\mathcal{J}^{r+m}\left(\overline{\mathbf{V}_{* *}((1+\mu) \mathbf{F})}\right)=\mathcal{J}^{r+m}\left(\overline{\mathbf{U}_{* *}((1+\mu) \mathbf{F})}\right) .
$$

Also, from Lemma 6, there exists $\lambda \in \bigoplus_{j \geq \kappa} \mathcal{P}_{j}^{\mathbf{t}}$ such that

$$
\left(1-\bar{\mu}_{\kappa}\right)\left(\mathbf{V}_{* *}((1+\mu) \mathbf{F})\right)=\mathbf{V}_{* *}((1+\lambda)(1+\mu) \mathbf{F})=\mathbf{V}_{* *}((1+v) \mathbf{F}),
$$

where we have defined $v:=(1+\lambda)(1+\mu)-1$. We observe that, as $\lambda_{\kappa}=-\bar{\mu}_{\kappa}$, then $\mathcal{J}^{\kappa-1}(v)=\mathcal{J}^{\kappa-1}(\mu)$, and

$$
v_{\kappa}=\mu_{\kappa}-\bar{\mu}_{\kappa}=\widetilde{\mu}_{\kappa} \in \widetilde{\mathcal{P}}_{\kappa}^{\mathbf{t}}
$$

Therefore $\mathcal{J}^{\mathcal{K}}(v) \in \bigoplus_{j=1}^{\kappa} \widetilde{\mathcal{P}}_{j}^{\mathbf{t}}$.

Moreover, as it is possible to extend the property showed in Lemma 1 item $\mathbf{g}$ ) for sum of vector fields and sum of scalar functions, we get

$$
\overline{\mathbf{V}_{* *}((1+v) \mathbf{F})}=\overline{\left(1-\bar{\mu}_{\kappa}\right)\left(\mathbf{V}_{* *}((1+\mu) \mathbf{F})\right)}=\left(1-\bar{\mu}_{\kappa}\right)\left(\overline{\mathbf{V}_{* *}((1+\mu) \mathbf{F})}\right)
$$

As $\mathcal{J}^{r+N-1}\left(\overline{\mathbf{V}_{* *}((1+v) \mathbf{F})}\right)=\mathbf{0}$, we get

$$
\mathcal{J}^{r+N}\left(\overline{\mathbf{V}_{* *}((1+v) \mathbf{F})}\right)=\mathcal{J}^{r+N}\left(\overline{\mathbf{V}_{* *}((1+\mu) \mathbf{F})}\right)=\mathcal{J}^{r+N}\left(\overline{\mathbf{U}_{* *}((1+\mu) \mathbf{F})}\right) .
$$

As $\mathcal{R}_{\kappa}^{\mathbf{t}}=\operatorname{Ker}\left(\ell_{\mathcal{\kappa}}\right) \widetilde{\mathbf{F}}_{r} \oplus \mathcal{R}_{\kappa}^{\mathbf{t}}$, we can write $\mathbf{V}_{\mathcal{K}}=\bar{\rho}_{\mathcal{\kappa}-r} \widetilde{\mathbf{F}}_{r}+\widetilde{\mathbf{V}}_{\mathcal{\kappa}}$, with $\bar{\rho}_{\mathcal{\kappa}-r} \in \operatorname{Ker}\left(\ell_{\mathcal{K}}\right)$ (for some $\rho_{\mathcal{\kappa}-r} \in \operatorname{Ker}\left(\ell_{\mathcal{K}}\right)$ ) and $\widetilde{\mathbf{V}}_{\mathcal{K}} \in \mathcal{R}_{\mathcal{K}}^{\mathrm{t}}$.

Moreover, since $\widetilde{\mathcal{P}}_{\mathcal{K}}^{\mathrm{t}}=\operatorname{Range}\left(\tilde{\ell}_{\mathcal{\kappa}}\right) \oplus \operatorname{Cor}\left(\tilde{\ell}_{\mathcal{\kappa}}\right)$, we can write $v_{\mathcal{\kappa}}=\nabla \bar{\eta}_{\kappa-r} \cdot \widetilde{\mathbf{F}}_{r}+\widetilde{v}_{\kappa}$, where $\bar{\eta}_{\mathcal{K}-r} \in \overline{\mathcal{P}}_{\mathcal{\kappa}-r}^{\mathbf{t}}$ and $\widetilde{v}_{\mathcal{K}} \in \operatorname{Cor}\left(\widetilde{\ell}_{\mathcal{K}}\right)$, we can assume without loss of generality that $\bar{\eta}_{\kappa-r} \in \overline{\mathcal{P}}_{\mathcal{\kappa}-r}^{\mathbf{t}} \backslash \operatorname{Ker}\left(\widetilde{\ell}_{\mathcal{\kappa}}\right)$ since otherwise $\nabla \bar{\eta}_{\kappa-r} \cdot \widetilde{\mathbf{F}}_{r}=0$.

Let us denote by $\widetilde{\Phi}$ and $\widetilde{\Psi}$ the transformations generated by $\mathbf{V}$ and $\left(\bar{\rho}_{\kappa-r}+\bar{\eta}_{\kappa-r}\right) \mathbf{F}$ respectively, and consider a generator $\mathbf{W}$ of the transformation $\widetilde{\Phi} \circ \widetilde{\Psi}^{-1}$. Then:

$$
\begin{aligned}
\mathbf{V}_{* *}((1+v) \mathbf{F}) & =\left(\widetilde{\Phi} \circ \widetilde{\Psi}^{-1} \circ \widetilde{\Psi}\right)_{*}((1+v) \mathbf{F})=\left(\widetilde{\Phi} \circ \widetilde{\Psi}^{-1}\right) *(\widetilde{\Psi} *((1+v) \mathbf{F})) \\
& =\mathbf{W}_{* *}\left(\left(\left(\bar{\rho}_{\mathcal{\kappa}-r}+\bar{\eta}_{\mathcal{\kappa}-r}\right) \mathbf{F}\right)_{* *}((1+v) \mathbf{F})\right) .
\end{aligned}
$$

From Lemma 8, there exists $\delta \in \bigoplus_{j \geq 1} \mathcal{P}_{j}^{\mathbf{t}}$ such that

$$
\mathbf{W}_{* *}\left(\left(\left(\bar{\rho}_{\kappa-r}+\bar{\eta}_{\kappa-r}\right) \mathbf{F}\right)_{* *}((1+v) \mathbf{F})\right)=\mathbf{W}_{* *}((1+\delta) \mathbf{F}),
$$

where $\delta$ and $v$ agree up to quasi-homogeneous degree $\kappa-1$ (i.e., $\mathcal{J}^{\kappa-1}(\delta)=\mathcal{J}^{\kappa-1}(v)$ ), and

$$
\delta_{\mathcal{K}}=v_{\mathcal{K}}-\nabla \bar{\rho}_{\kappa-r} \cdot \widetilde{\mathbf{F}}_{r}-\nabla \bar{\eta}_{\kappa-r} \cdot \widetilde{\mathbf{F}}_{r}=\nabla \bar{\eta}_{\kappa-r} \cdot \widetilde{\mathbf{F}}_{r}+\widetilde{v}_{\mathcal{K}}-\nabla \bar{\eta}_{\kappa-r} \cdot \widetilde{\mathbf{F}}_{r}=\widetilde{v}_{\mathcal{\kappa}} \in \operatorname{Cor}\left(\widetilde{\ell}_{\kappa}\right) .
$$

Therefore $\mathcal{J}^{\kappa}(\delta) \in \bigoplus_{j=1}^{\kappa} \operatorname{Cor}\left(\tilde{\ell}_{j}\right)$.

On the other hand, we get that $-\left(\bar{\rho}_{\kappa-r}+\bar{\eta}_{\kappa-r}\right) \widetilde{\mathbf{F}}_{r}+\cdots$ is a generator of the transformation $\widetilde{\Psi}^{-1}$. Consequently, from Lemma 7, we have $\mathcal{J}^{\kappa-1}(\mathbf{W})=\mathcal{J}^{\kappa-1}(\mathbf{V})$, and taking into account that $\bar{\eta}_{\mathcal{K}-r} \in \overline{\mathcal{P}}_{\mathcal{K}-r}^{\mathbf{t}} \backslash \operatorname{Ker}\left(\widetilde{\ell}_{\mathcal{K}}\right)$ we get:

$$
\mathbf{W}_{\kappa}=\mathbf{V}_{\kappa}-\left(\bar{\rho}_{\kappa-r}+\bar{\eta}_{\kappa-r}\right) \widetilde{\mathbf{F}}_{r}=\bar{\rho}_{\kappa-r} \widetilde{\mathbf{F}}_{r}+\widetilde{\mathbf{V}}_{\kappa}-\bar{\rho}_{\kappa-r} \widetilde{\mathbf{F}}_{r}-\bar{\eta}_{\kappa-r} \widetilde{\mathbf{F}}_{r}=\widetilde{\mathbf{V}}_{\kappa}-\bar{\eta}_{\kappa-r} \widetilde{\mathbf{F}}_{r} \in \mathcal{R}_{\kappa}^{\mathbf{t}} .
$$

Therefore

$$
\mathbf{W}_{* *}((1+\delta) \mathbf{F})=\mathbf{V}_{* *}((1+v) \mathbf{F}) .
$$


In summary, from the generators $(\mathbf{U}, \mu)$ with $\mathcal{J}^{\kappa-1}(\mathbf{U}) \in \bigoplus_{j=1}^{\kappa-1} \mathcal{Q}_{j}^{\mathbf{t}}$ and $\mathcal{J}^{\kappa-1}(\mu)=$ $\bigoplus_{j=1}^{\kappa-1} \mathcal{P}_{j}^{\mathbf{t}}$ we have found others $(\mathbf{V}, v)$ such that $\mathcal{J}^{\mathcal{K}-1}(\mathbf{V})=\mathcal{J}^{\kappa-1}(\mathbf{U}), \mathbf{V}_{\kappa} \in \widetilde{\mathcal{Q}}_{\kappa^{\prime}}^{\mathbf{t}}, \mathcal{J}^{\kappa-1}(v)=$ $\mathcal{J}^{\kappa-1}(\mu), v_{\kappa} \in \widetilde{\mathcal{P}}_{\kappa}^{\mathbf{t}}$ and $\mathcal{J}^{r+N}\left(\overline{\mathbf{V}_{* *}((1+v) \mathbf{F})}\right)=\mathcal{J}^{r+N}\left(\overline{\mathbf{U}_{* *}((1+\mu) \mathbf{F})}\right)$. From the generators $(\mathbf{V}, v)$ we have found others $(\mathbf{W}, \delta)$ such that $\mathcal{J}^{\kappa}(\mathbf{W}) \in \bigoplus_{j=1}^{\kappa} \mathcal{R}_{j}^{\mathrm{t}}, \mathcal{J}^{\mathcal{K}}(\delta)=$ $\bigoplus_{j=1}^{\kappa} \operatorname{Cor}\left(\widetilde{\ell}_{j}\right)$ and $\mathbf{W}_{* *}((1+\delta) \mathbf{F})=\mathbf{V}_{* *}((1+v) \mathbf{F})$. Therefore $\mathcal{J}^{r+\kappa}\left(\overline{\mathbf{W}_{* *}((1+\delta) \mathbf{F})}\right)=$ $\mathcal{J}^{r+\kappa}\left(\overline{\mathbf{U}_{* *}((1+\mu) \mathbf{F})}\right)$, so the value of $\kappa$ for these new temporal and spatial generators is greater than the previous value. Taking $\mathbf{W}$ and $\delta$ as new $\mathbf{U}$ and $\mu$, this procedure continues until the statement is proven.

Proof. [Statement b) of Theorem 2] If $\widetilde{g}_{i}=0, \bar{\eta}_{i}=0$ for all $i>r$ then the system (5) is reversible to $x$, therefore system $\dot{\mathbf{x}}=\mathbf{F}(\mathbf{x})$ is orbitally $R_{x}$-reversible and the sufficiency conditions follows.

We prove the necessity. Let $N=\min \left\{i \in \mathbb{N}: \widetilde{g}_{r+i} \neq 0\right.$ or $\left.\bar{\eta}_{r+i} \neq 0\right\}$. On the other hand $\mathbf{F}$ is orbitally $R_{x}$-reversible, then by applying Theorem 1 there exists $\mathbf{V}=\sum_{j \geq 1} \mathbf{V}_{j}$, $\mathbf{V}_{j} \in \mathcal{Q}_{j}^{\mathbf{t}}, v=\sum_{j \geq 1} v_{j}, v_{j} \in \mathcal{P}_{j}^{\mathbf{t}}$ and $\Phi_{0} \in \mathcal{Q}_{0}^{\mathbf{t}}$ such that $\mathbf{V}_{* *}\left((1+v)\left(\Phi_{0}\right)_{*} \mathbf{F}\right)$ is $R_{x}$-reversible. As $\mathbf{F}=\widetilde{\mathbf{F}}_{r}+\cdots$, we can take $\Phi_{0}=I d$. In particular $\mathcal{J}^{r+N-1}\left(\overline{\mathbf{V}_{* *}((1+v) \mathbf{F})}\right)=\mathbf{0}$. By applying Proposition 3 there exists $\mathbf{W}=\sum_{j \geq 1} \mathbf{W}_{j}, \mathbf{W}_{j} \in \mathcal{Q}_{j}^{\mathbf{t}}$, such that $\mathcal{J}^{N}(\mathbf{W}) \in$ $\bigoplus_{j=1}^{N} \mathcal{R}_{j}^{\mathbf{t}}$ and $\delta=\sum_{j \geq 1} \delta_{j}, \delta_{j} \in \mathcal{P}_{j}^{\mathbf{t}}$ such that $\mathcal{J}^{N}(\delta) \in \bigoplus_{j=1}^{N} \operatorname{Cor}\left(\tilde{\ell}_{j}\right)$ verifying

$$
\mathcal{J}^{r+N}\left(\overline{\mathbf{W}_{* *}((1+\delta) \mathbf{F})}\right)=\mathcal{J}^{r+N}\left(\overline{\mathbf{V}_{* *}((1+v) \mathbf{F})}\right) .
$$

Therefore $\mathcal{J}^{r+N-1}\left(\overline{\mathbf{W}_{* *}((1+\delta) \mathbf{F})}\right)=\mathbf{0}$.

Consider the spatial generator $\widetilde{\mathbf{U}}=\sum_{i \geq 1} \widetilde{\mathbf{U}}_{i}$ given in (5). We will prove that $\widetilde{\mathbf{U}}_{i}=\mathbf{W}_{i}$ for $1 \leq i<N$ which will lead us to a contradiction.

- $\quad$ Case $i=1, N>1$. In this case

$\overline{\mathcal{L}}_{r+1}\left(\widetilde{\mathbf{U}}_{1}, \widetilde{\mu}_{1}\right)=-\widetilde{\mathbf{U}}_{* *}((1+\widetilde{\mu}) \mathbf{F})_{r+1}=\mathbf{0}=-\overline{\mathbf{W}}_{* *}((1+\delta) \mathbf{F})_{r+1}=\overline{\mathcal{L}}_{r+1}\left(\mathbf{W}_{1}, \delta_{1}\right)$.

Notice that $\mathbf{W}_{1} \in \widetilde{\mathcal{Q}}_{1}^{\mathbf{t}}$ and $\delta_{1} \in \operatorname{Cor}\left(\widetilde{\ell}_{1}\right)$. Therefore $\overline{\mathcal{L}}_{r+1}\left(\widetilde{\mathbf{U}}_{1}-\mathbf{W}_{1}, \widetilde{\mu}_{1}-\delta_{1}\right)=\mathbf{0}$, but by Proposition $2 \operatorname{Ker}\left(\overline{\mathcal{L}}_{r+1}\right)=\{\mathbf{0}\}$, then we get $\widetilde{\mathbf{U}}_{1}=\mathbf{W}_{1}, \widetilde{\mu}_{1}=\delta_{1}$.

- $\quad$ Case $N>1$ and we suppose that $\widetilde{\mathbf{U}}_{i}=\mathbf{W}_{i}, \widetilde{\mu}_{i}=\delta_{i}$ for $1 \leq i<i_{0}$, with $i_{0}<N$. In this case $\left(\left(\sum_{j=1}^{i_{0}-1} \widetilde{\mathbf{U}}_{j}\right)_{* *}\left(\left(1+\sum_{j=1}^{i_{0}-1} \widetilde{\mu}_{j}\right) \mathbf{F}\right)\right)_{r+i_{0}}=\left(\left(\sum_{j=1}^{i_{0}-1} \mathbf{W}_{j}\right)_{* *}\left(\left(1+\sum_{j=1}^{i_{0}-1} \delta_{j}\right) \mathbf{F}\right)\right)_{r+i_{0}}$.

On the other hand

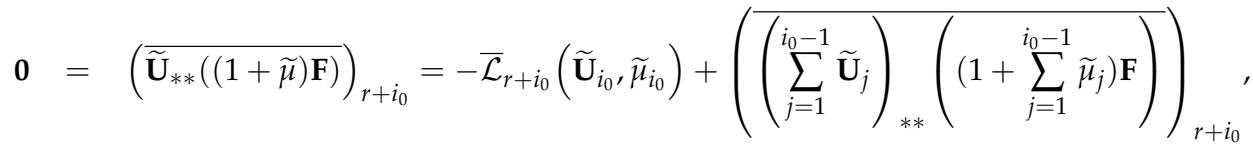

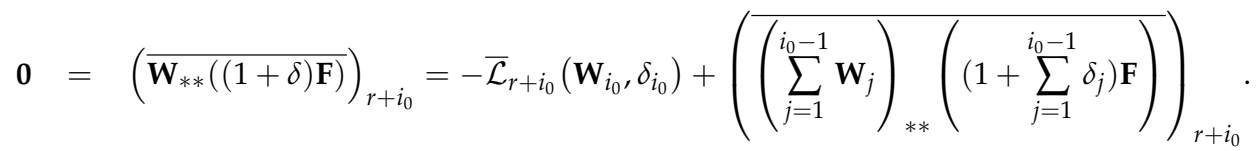

Notice that $\mathbf{W}_{i_{0}} \in \widetilde{\mathcal{Q}}_{i_{0}}^{\mathbf{t}}$ and $\delta_{i_{0}} \in \operatorname{Cor}\left(\widetilde{\ell}_{i_{0}}\right)$. Therefore $\overline{\mathcal{L}}_{r+i_{0}}\left(\widetilde{\mathbf{U}}_{i_{0}}-\mathbf{W}_{i_{0}}, \widetilde{\mu}_{i_{0}}-\delta_{i_{0}}\right)=\mathbf{0}$, but by Proposition $2 \operatorname{Ker}\left(\overline{\mathcal{L}}_{r+i_{0}}\right)=\{\mathbf{0}\}$, then we get $\widetilde{\mathbf{U}}_{i_{0}}=\mathbf{W}_{i_{0}}, \widetilde{\mu}_{i_{0}}=\delta_{i_{0}}$. 
Reasoning in this way we would obtain that $\widetilde{\mathbf{U}}_{i}=\mathbf{W}_{i}$ and $\widetilde{\mu}_{i}=\delta_{i}$ for $1 \leq i<N$ and then

$$
\begin{aligned}
& \left(\left(\sum_{j=1}^{N-1} \widetilde{\mathbf{U}}_{j}\right)_{* *}\left(\left(1+\sum_{j=1}^{N-1} \widetilde{\mu}_{j}\right) \mathbf{F}\right)\right)_{r+N}=\left(\left(\sum_{j=1}^{N-1} \mathbf{W}_{j}\right)_{* *}\left(\left(1+\sum_{j=1}^{N-1} \delta_{j}\right) \mathbf{F}\right)\right)_{r+N} \text {. So }
\end{aligned}
$$

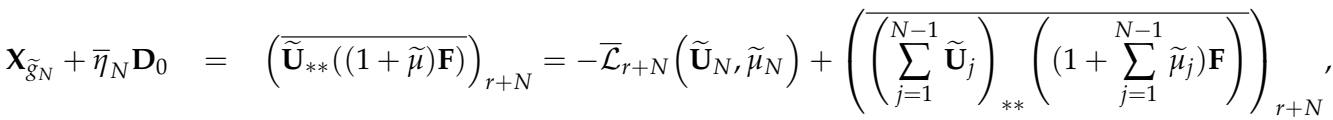

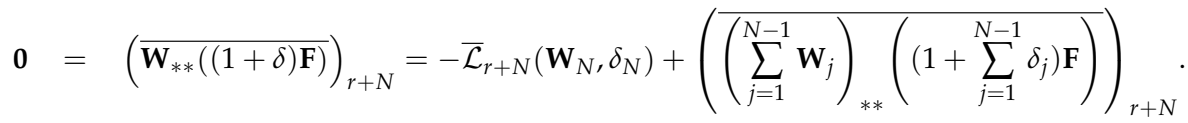

Notice that $\mathbf{W}_{N} \in \widetilde{\mathcal{Q}}_{N}^{\mathbf{t}}$ and $\delta_{N} \in \operatorname{Cor}\left(\widetilde{\ell}_{N}\right)$. Therefore $\mathbf{X}_{\widetilde{g}_{N}}+\bar{\eta}_{N} \mathbf{D}_{0}=\overline{\mathcal{L}}_{r+N}\left(\mathbf{W}_{N}-\right.$ $\left.\widetilde{\mathbf{U}}_{N}, \delta_{N}-\widetilde{\mu}_{N}\right)$. In consequence, $\mathbf{X}_{\widetilde{g}_{N}}+\bar{\eta}_{N} \mathbf{D}_{0} \in \operatorname{Range}\left(\overline{\mathcal{L}}_{r+N}\right) \cap \operatorname{Cor}\left(\overline{\mathcal{L}}_{r+N}\right)$. Therefore $\mathbf{X}_{\widetilde{g}_{N}}+\bar{\eta}_{N} \mathbf{D}_{0}=\mathbf{0}$ and then $\widetilde{g}_{N}=\bar{\eta}_{N}=0$, which is contradictory.

\section{Orbital $R_{y}$-Reversibility}

The following result provides a necessary and sufficient condition to have an orbital $R_{y}$-reversible vector field whose first quasi-homogenous component is $R_{y}$-reversible.

Theorem 4. Let $\mathbf{F}:=\mathbf{F}_{r}+\cdots$ be the vector field (3) such that $\widetilde{\mathbf{F}}_{r}$ is $R_{y}$-reversible. If $\operatorname{Ker}\left(\widetilde{\ell}_{r+k+|\mathbf{t}|}^{\mathrm{c}}\right)$ $=\{0\}$ for all $k \in \mathbb{N}$ then

(a) There exist $\widetilde{\mathbf{U}}=\sum_{k \geq 1} \widetilde{\mathbf{U}}_{k}, \widetilde{\mathbf{U}}_{k} \in \mathcal{R}_{k}^{\mathbf{t}}$ and $\widetilde{\mu}=\sum_{k \geq 1} \widetilde{\mu}_{k}, \widetilde{\mu}_{k} \in \operatorname{Cor}\left(\widetilde{\ell}_{k}\right)$ such that

$$
\widetilde{\mathbf{U}}_{* *}((1+\widetilde{\mu}) \mathbf{F})=\widetilde{\mathbf{F}}_{r}+\left(\begin{array}{c}
y F\left(x, y^{2}\right) \\
G\left(x, y^{2}\right)
\end{array}\right)+\sum_{k \geq 1}\left(\mathbf{x}_{\widetilde{g}_{r+k+|t|}}+\bar{\eta}_{r+k} \mathbf{D}_{0}\right),
$$

where $\bar{\eta}_{r+k} \in \operatorname{Cor}\left(\bar{\ell}_{r+k}\right), \widetilde{g}_{r+k+|\mathbf{t}|} \in \operatorname{Cor}\left(\widetilde{\ell}_{r+k+|\mathbf{t}|}^{\mathrm{c}}\right)$ and $\left(y F\left(x, y^{2}\right), G\left(x, y^{2}\right)\right)^{T}$ is a vector field sum of quasi-homogeneous vector fields of type $\mathbf{t}$ and degree greater than $r$. Notice that the notations $\widetilde{\mathbf{U}}, \widetilde{\mu}, \bar{\ell}_{k}, \widetilde{\ell}_{r+k}, \widetilde{\ell}_{r+k+|\mathbf{t}|}^{\mathrm{c}}$ have the same meaning as those used in Theorem $2 \mathrm{but}$ applied to variable $y$ instead of variable $x$. In this case the distinguished variable is $y$.

(b) System (7) is orbitally $R_{y}$-reversible if, and only if, $\bar{g}_{r+k+|\mathbf{t}|}=0, \bar{\eta}_{r+k}=0$ for all $k>0$.

Proof. The proof follows the same steps as the proof of Theorem 2 since symmetry properties are verified either with respect to $x$ or with respect to $y$.

The following result and the previous Proposition 1 provide a tool to calculate the corranges that appear in the previous Theorems regardless of whether the distinguished variable is the variable $x$ or $y$.

Proposition 4. Let be the following linear operators:

$$
\begin{aligned}
& \ell_{r+k+|\mathbf{t}|}^{\mathrm{c}}: \Delta_{k+|\mathbf{t}|} \longrightarrow \Delta_{r+k+|\mathbf{t}|} \bar{\ell}_{r+k+|\mathbf{t}|}^{\mathrm{c}}: \widetilde{\Delta}_{k+|\mathbf{t}|} \longrightarrow \bar{\Delta}_{r+k+|\mathbf{t}|} \\
& g \rightarrow \operatorname{Proy}_{\Delta_{r+k+|t|}}\left(\nabla g \cdot \widetilde{\mathbf{G}}_{r}\right), \quad g \rightarrow \operatorname{Proy}_{\bar{\Delta}_{r+k+|t|}}\left(\nabla g \cdot \widetilde{\mathbf{G}}_{r}\right), \\
& \text { where } \Delta_{k+|\mathbf{t}|}=\widetilde{\Delta}_{k+|\mathbf{t}|} \oplus \bar{\Delta}_{k+|\mathbf{t}|} \text { and } \widetilde{\mathbf{G}}_{r}=\widetilde{\mathbf{F}}_{r}-\frac{r+|\mathbf{t}|}{i+|\mathbf{t}|} \mu \mathbf{D}_{0} \text {. Then } \operatorname{Cor}\left(\widetilde{\ell}_{r+k+|\mathbf{t}|}^{\mathrm{c}}\right) \oplus \operatorname{Cor}\left(\bar{\ell}_{r+k+|\mathbf{t}|}^{\mathrm{c}}\right) \\
& \text { is a complementary subspace of Range }\left(\ell_{r+k+|\mathbf{t}|}^{c}\right) \text {. }
\end{aligned}
$$

Proof. Notice that we have $\mu=\frac{1}{r+|\mathbf{t}|} \operatorname{div}\left(\widetilde{\mathbf{F}}_{r}\right)(\mu$ defined in (4)), and by Lemma 1 item o) $\mu \in \widetilde{\mathcal{P}}_{r}^{\mathbf{t}}$, then $\mu \mathbf{D}_{0} \in \widetilde{\mathcal{Q}}_{r}^{\mathbf{t}}\left(\mathbf{D}_{0}\right.$ defined in (4)). So, if we considered $\widetilde{\mathbf{G}}_{r}:=\widetilde{\mathbf{F}}_{r}-\frac{r+|\mathbf{t}|}{i+|\mathbf{t}|} \mu \mathbf{D}_{0}$, then $\widetilde{\mathbf{G}}_{r} \in \widetilde{\mathcal{Q}}_{r}^{\mathbf{t}}$. Therefore $\ell_{r+k+|\mathbf{t}|}^{\mathrm{c}} \mid$ is the Lie-derivative operator of $\widetilde{\mathbf{G}}_{r}$ restricted to $\Delta_{k+|\mathbf{t}|}$. 
As $\Delta_{k+|\mathbf{t}|}=\widetilde{\Delta}_{k+|\mathbf{t}|} \oplus \bar{\Delta}_{k+|\mathbf{t}|}$ and, by Lemma 1 items $\mathbf{h}$ ) and $\mathbf{j}$ ), the matrix of operator $\ell_{r+k+|\mathbf{t}|}^{\mathrm{c}}$ can be expressed as

\begin{tabular}{|c|c|c|}
\hline$\widetilde{\Delta}_{k+|\mathbf{t}|}^{\mathbf{t}}$ & $\bar{\Delta}_{k+|\mathbf{t}|}^{\mathbf{t}}$ & \\
\hline $\bar{\ell}_{r+k+|\mathbf{t}|}^{\mathrm{c}}\left(\widetilde{\Delta}_{k+|\mathbf{t}|}\right)$ & $\mathbf{0}$ & $\bar{\Delta}_{r+k+|\mathbf{t}|}^{\mathbf{t}}$ \\
\hline $\mathbf{0}$ & $\widetilde{\ell}_{r+k+|\mathbf{t}|}^{\mathrm{c}}\left(\bar{\Delta}_{k+|\mathbf{t}|}\right)$ & $\widetilde{\Delta}_{r+k+|\mathbf{t}|}^{\mathbf{t}}$ \\
\hline
\end{tabular}

The matrices of the reduced operator $\widetilde{\ell}_{i+|\mathbf{t}|}^{\mathrm{c}}$ and $\bar{\ell}_{i+|\mathbf{t}|}^{\mathrm{c}}$ are submatrices of the matrix of $\ell_{i+|\mathbf{t}|}^{\mathrm{c}}$ and the result is obtained.

The following results will be useful in the computation of orbitally reversible families.

Lemma 9. Let $\mathbf{F}:=\mathbf{F}_{r}+\cdots$ be a vector field such that $\mathbf{F}_{r}=\mathbf{X}_{h}$ and $h$ has only simple factors in its decomposition on $\mathbb{C}[x, y]$, then $\operatorname{Ker}\left(\widetilde{\ell}_{r+k+|\mathbf{t}|}^{c}\right)=\{0\}$ for all $k \in \mathbb{N}$.

Proof. Suppose that there exist $k \in \mathbf{N}$ such that $\operatorname{Ker}\left(\widetilde{\ell}_{r+k+|\mathbf{t}|}^{\mathrm{c}}\right) \neq\{0\}$, by proof of Proposition 4 there exists $g \in \bar{\Delta}_{k+|\mathbf{t}|}$ such that $\ell_{r+k+|\mathbf{t}|}^{c}(g) \in\langle h\rangle$. In this case, i.e., $\mathbf{F}_{r}=\mathbf{X}_{h}$, is $\ell_{r+k+|\mathbf{t}|}^{c}(g)=\ell_{r+k+|\mathbf{t}|}(g)$. So $\ell_{r+k+|\mathbf{t}|}(g) \in\langle h\rangle$. As $h$ has simple factor $h=\prod_{i=1}^{s} f_{i}$, where each $f_{i}$ is a simple factor, therefore $\mathbf{X}_{h}$ is irreducible and $\ell_{r+k+|\mathbf{t}|}(g) \in\left\langle f_{i}\right\rangle$ for all $1 \leq i \leq s$. By [40] [Lemma 3.21] we get $g \in\left\langle f_{i}\right\rangle$ and then $g \in\langle h\rangle \cap \bar{\Delta}_{k^{\prime}+|\mathbf{t}|}$ which is a contradiction.

Proposition 5. Let $H=h+\cdots$ with $h \in \mathcal{P}_{s}^{\mathbf{t}}, \Phi$ a change of variable and $G=H \circ \Phi$, then $\mathbf{X}_{G}$ is orbitally equivalent to $\mathbf{X}_{H}$.

Proof. It is enough to see that $\mathbf{X}_{G}=\mathbf{X}_{H \circ \Phi}=(\operatorname{det} D \Phi) \Phi_{*} \mathbf{X}_{H}$.

\section{Applications}

The results of Theorem 2 allow to build an algorithm for the computation of the necessary conditions for a vector field within a family of vector fields to be orbitally $R_{x^{-}}$ reversible. (Analogously with the Theorem 4 for the orbitally $R_{y}$-reversibility). In this section we apply these algorithms to detect vector fields that are orbitally reversible within a family of vector fields.

We are interested in studying the orbital reversibility of certain families of monodromic systems and thereby determine some families of systems with a center at the origin.

Example 1. First we come back to the example of the simple pendulum swing without friction. The movement equation is $\ell \ddot{\theta}+g \sin \theta=0$ where $\ell$ is the length of the pendulum, $g$ is acceleration due to gravity and $\theta$ is the angular displacement. Using the small-angle approximation $\sin \theta \approx \theta$ we arrive to the equation of the harmonic oscillator $\ell \ddot{\theta}+g \theta=0$. Scaling this equation and renaming the new variable as $x$ we get $\ddot{x}+x=0$ that can be transformed into the differential system $\dot{x}=y$, $\dot{y}=-x$ introducing the new variable $y=\dot{x}$. This system has a global center at the origin and all the phase portrait is foliated by periodic orbits. Moreover this differential system has the time-reversal symmetry, in fact is $R_{x}$-reversible and $R_{y}$-reversible. It is true that, in general, the presence of friction breaks the time-reversal symmetry but sometimes this is not true and in fact it depends on the way we model the friction. A first approximation to the friction is to consider that is proportional to velocity adding the term $k \dot{\theta}$ where $k$ is the friction constant. In this case the differential equation takes the form $\ell \ddot{\theta}+k \dot{\theta}+g \theta=0$. This equation doing the same rescaling that before is transformed to $\ddot{x}+k \dot{x}+x=0$ or into the differential system $\dot{x}=y, \dot{y}=-k y-x$. This system for values of $k \neq 0$ has a focus at the origin and consequently has not a time-reversal symmetry. However we can consider generalizations of the friction term that must be determined according with the values obtained from the experimentation of the model. 
Van der Pol studied an electrical circuit with a vacuum tube, in fact with a triode, and arrived to an equation of the form $\ddot{x}+\mu\left(x^{2}+1\right) \dot{x}+x=0$, see for instance [41]. Here the friction term is given by $\mu\left(x^{2}+1\right) \dot{x}$. During the development of radio and vacuum tube technology, Liénard [42] showed the existence and uniqueness of a limit cycle for Van der Pol equation and established a generalization of the Van der Pol equation given by

$$
\ddot{x}+f(x) \dot{x}+x=0,
$$

where $f(x)$ is an analytic function called the damping of the Liénard equation. This Liénard equation can be rewritten into the differential system $\dot{x}=y, \dot{x}=-x-y f(x)$. By means of the Liénard transformation $y \rightarrow y+F(x)$ where $F(x)=\int_{0}^{x} f(x) d x$, this last system can be written as

$$
\dot{x}=y-F(x), \quad \dot{y}=-x .
$$

Inside such family of differential systems there are systems that admit a time-reversal symmetry. If we apply the method developed in this work we can arrive to the following theorem.

Theorem 5. System $\dot{x}=y-F(x), \dot{y}=-x$ is orbitally reversible if, and only if, $F(x)=\Phi\left(x^{2}\right)$. Moreover under this condition the system has a center at the origin.

In fact such result can be generalized for differential systems of the form $\dot{x}=y-$ $F(x), \quad \dot{y}=-g(x)$ where $g$ is an analytic function with $g(0)=0$ and $g^{\prime}(0)>0$, see for instance $[30,41]$ and references therein. Consequently when we have damping or some type of friction we still can have a time-reversal symmetry that implies a specular symmetric of the phase portrait. However this symmetry can be hidden because we have not our system in the normal form that reveals the existence of such symmetry.

This analysis can be applied to more general differential systems as the following examples show.

Example 2. Consider the differential system whose vector field is the sum of two quasi-homogenous fields of degrees 7 and 8 respectively with respect to type $\mathbf{t}=(2,3)$.

$$
\left(\begin{array}{c}
\dot{x} \\
\dot{y}
\end{array}\right)=\overbrace{\left(\begin{array}{c}
y^{3}+2 a x^{3} y \\
-x^{5}-3 a x^{2} y^{2}
\end{array}\right)}^{\mathrm{F}_{7}}+\overbrace{\left(\begin{array}{c}
a_{50} x^{5}+a_{22} x^{2} y^{2} \\
b_{41} x^{4} y+b_{13} x y^{3}
\end{array}\right)}^{\mathrm{F}_{8}},|a| \neq \frac{1}{\sqrt{6}} .
$$

The origin of system (8) is monodromic, see [24] [Lemma 4.37], therefore the origin of these fields are possible centers. The analytical integrability of system (8) has been studied in [24]. Here we are interested in studying the orbital reversibility and thereby to determine degenerate centers in this family.

In order to study the orbital reversibility of the family (8), we first compute the possible reversibilities of the first component $\mathbf{F}_{7}$ (modulo a zero-degree change of variables).

Proposition 6. The quasi-homogeneous vector field $\mathbf{F}_{7}$ given in (8) is $R_{x}$-reversible if $a=0$ and $\mathbf{F}_{7}$ is $R_{y}$-reversible for all $a \in \mathbb{R}$ and they are the only reversibilities of $\mathbf{F}_{7}$ (modulo a zero-degree change of variables).

Proof. For the type $\mathbf{t}=(2,3)$ we get $\mathcal{Q}_{0}^{\mathbf{t}}=\left\{\left(a_{10} x, b_{01} y\right)^{T}: a_{10}, b_{01} \in \mathbb{R}\right\}$, then $\left(\Phi_{0}\right)_{*} \mathbf{F}_{7}$ is $\mathrm{R}_{x}$-reversible if, and only if, $a=0$, and $\left(\Phi_{0}\right)_{*} \mathbf{F}_{7}$ is $\mathrm{R}_{y}$-reversible for all $\Phi_{0} \in \mathcal{Q}_{0}^{\mathrm{t}}$. Therefore, these two reversibilities are the only reversibilities of $\mathbf{F}_{7}$ modulo a zero degree change of variables.

Remark 1. As a consequence of the previous result we only have to analyze the orbital $R_{x^{-}}$ reversibility of system (8) for $a=0$ and the orbital $R_{y}$-reversibility for $a \in \mathbb{R}$. 
Theorem 6. System (8) is orbitally reversible if, and only if, one of following conditions holds.

(a) $5 a_{50}+b_{41}=2 a_{22}+3 b_{13}=0$.

(b) $a=b_{41}=a_{50}=0$.

Proof. By Remark 1 we have to study only the orbital $R_{x}$ - or $R_{y}$-reversibility. The first quasi-homogeneous term of system (8) is $\mathbf{F}_{7}=\mathbf{X}_{h} \in \mathcal{Q}_{7}^{\mathbf{t}}$ with $\mathbf{t}=(2,3)$ and $h=-\left(2 x^{6}+\right.$ $\left.12 a x^{3} y^{2}+3 y^{4}\right) / 12$.

If $6 a^{2} \neq 1, h$ has only simple factors in its decomposition on $\mathbb{C}[x, y]$, so by Lemma 9 , $\operatorname{Ker}\left(\tilde{\ell}_{r+k+|\mathbf{t}|}^{\mathrm{c}}\right)=\{0\}$ for all $k \in \mathbb{N}$ and we can apply Theorems 2 and 4 .

We first study the orbital $R_{x}$-reversibility in the case $a=0$. Applying Theorem 2 statement (a), a normal form of system (8) is

$$
\left(\begin{array}{c}
\dot{x} \\
\dot{y}
\end{array}\right)=\mathbf{F}_{7}+\left(\begin{array}{c}
F\left(x^{2}, y\right) \\
x G\left(x^{2}, y\right)
\end{array}\right)+\sum_{k \geq 1}\left(\mathbf{x}_{\widetilde{g}_{12+k}}+\bar{\eta}_{7+k} \mathbf{D}_{0}\right),
$$

where $\tilde{g}_{12+k} \in \operatorname{Cor}\left(\widetilde{\ell}_{12+k}^{c}\right)$ and $\bar{\eta}_{7+k} \in \operatorname{Cor}\left(\bar{\ell}_{7+k}\right)$. So, by Theorem 2 statement (b), system (8) is orbitally $R_{x}$-reversible if, and only if, $\widetilde{g}_{12+k}=\bar{\eta}_{7+k}=0$ for $k \in \mathbb{N}$.

In order to calculate the first terms in the normal form that prevent the orbital reversibility of system (8), we need to compute the first four non-reversible terms and for this we need to determine $\operatorname{Cor}\left(\ell_{7+k}\right)$ and $\operatorname{Cor}\left(\ell_{12+k}^{c}\right)$ for $1 \leq k \leq 4$.

- $\quad \widetilde{\mathcal{P}}_{1}^{\mathbf{t}}=\{0\}$ and $\overline{\mathcal{P}}_{8}^{\mathbf{t}}=\operatorname{span}\left\{x^{4}\right\}$, therefore $\operatorname{Cor}\left(\bar{\ell}_{8}\right)=\operatorname{span}\left\{x^{4}\right\}$.

- $\bar{\Delta}_{6}=\{0\}$ and $\widetilde{\Delta}_{13}=\{0\}$, therefore $\operatorname{Cor}\left(\widetilde{\ell}_{13}^{\mathrm{c}}\right)=\{0\}$.

- $\quad \widetilde{\mathcal{P}}_{2}^{\mathbf{t}}=\operatorname{span}\{x\}$ and $\overline{\mathcal{P}}_{9}^{\mathbf{t}}=\operatorname{span}\left\{y^{3}\right\}$. If we take $p(x, y)=a_{10} x \in \widetilde{\mathcal{P}}_{1}^{\mathbf{t}}$, then $\ell_{9}(p)=$ $a_{10} y^{3}$ and therefore $\operatorname{Cor}\left(\bar{\ell}_{9}\right)=\{0\}$.

- $\bar{\Delta}_{7}=\operatorname{span}\left\{x^{2} y\right\}$ and $\widetilde{\Delta}_{14}=\operatorname{span} x^{7}$. If we take $g(x, y)=c_{21} x^{2} y \in \bar{\Delta}_{7}^{\mathbf{t}}$, then $\widetilde{\ell}_{14}^{\mathrm{c}}(g)=$ $2 c_{21} x y^{4}-c_{21} x^{7}=-\frac{7}{3} c_{21} x^{7}-\frac{8}{3} c_{21} h$ and therefore $\operatorname{Cor}\left(\widetilde{\ell}_{14}^{c}\right)=\{0\}$.

- $\quad \widetilde{\mathcal{P}}_{3}^{\mathbf{t}}=\operatorname{span}\{0\}$ and $\overline{\mathcal{P}}_{10}^{\mathbf{t}}=\operatorname{span}\left\{x^{2} y^{2}\right\}$, therefore $\operatorname{Cor}\left(\bar{\ell}_{10}\right)=\operatorname{span}\left\{x^{2} y^{2}\right\}$.

- $\bar{\Delta}_{8}=\operatorname{span}\left\{x^{4}\right\}$ and $\widetilde{\Delta}_{15}=\operatorname{span} x^{3} y^{3}$. If we take $g(x, y)=c_{40} x^{4} \in \bar{\Delta}_{8}^{\mathbf{t}}$, then $\widetilde{\ell}_{15}^{\mathrm{c}}(g)=$ $4 c_{40} x^{3} y^{3}$ and therefore $\operatorname{Cor}\left(\widetilde{\ell}_{15}^{c}\right)=\{0\}$.

- $\quad \widetilde{\mathcal{P}}_{4}^{\mathbf{t}}=\operatorname{span}\{0\}$ and $\overline{\mathcal{P}}_{11}^{\mathbf{t}}=\operatorname{span}\left\{x^{4} y\right\}$, therefore $\operatorname{Cor}\left(\bar{\ell}_{11}\right)=\operatorname{span}\left\{x^{4} y\right\}$.

- $\bar{\Delta}_{9}=\operatorname{span}\left\{y^{3}\right\}$ and $\widetilde{\Delta}_{16}=\operatorname{span} x^{5} y^{2}$. If we take $g(x, y)=c_{03} y^{3} \in \bar{\Delta}_{9}^{\mathbf{t}}$, then $\widetilde{\ell}_{15}^{\mathrm{c}}(g)=$ $-3 c_{40} x^{5} y^{2}$ and therefore $\operatorname{Cor}\left(\widetilde{\ell}_{15}^{c}\right)=\{0\}$.

So, $\bar{\eta}_{8}=\alpha_{8} x^{4}, \bar{\eta}_{9}=0, \bar{\eta}_{10}=\alpha_{10} x^{2} y^{2}, \bar{\eta}_{11}=\alpha_{11} x^{4} y$ and $\widetilde{g}_{12+k}=0$ for $1 \leq k \leq 4$.

Calculating the first coefficient of the normal form, we obtain $\alpha_{8}=\frac{1}{13}\left(5 a_{50}+b_{41}\right)=0$. Imposing this condition, i.e., $b_{41}=-5 a_{50}$, the second coefficient is given by

$$
\alpha_{10}=\frac{1}{35}\left(a_{50}\left(2 a_{22}+3 b_{13}\right)\left(5 a_{22}-3 b_{13}\right)\right) .
$$

(i) If $2 a_{22}+3 b_{13}=0$ we get a particular case of item (a). In this case system (8) is Hamiltonian with Hamiltonian function $H(x, y)=-\left(\frac{1}{6} x^{6}+a x^{3} y^{2}+\frac{1}{4} y^{4}+a_{50} x^{5} y+\right.$ $\left.\frac{1}{3} a_{22} x^{2} y^{3}\right)$. There exists a change of variables of the form identity plus non linear terms $\Phi$, such that $G(x, y):=\mathbf{H} \circ \Phi(x, y)=-\left(\frac{1}{6} x^{6}+a x^{3} y^{2}+\frac{1}{4} y^{4}+\beta_{9} x^{4} y^{2}\right)$, see [43] [Theorem 4]. By Proposition 5 we have that $\mathbf{X}_{H}$ is orbitally equivalent to $\mathbf{X}_{G}$. Finally, taking into account that $G(x,-y)=G(x, y)$, system (8) is $R_{y}$-orbitally reversible. 
(ii) If $a_{50}=0$ we get case $\mathbf{b}$ ) and system (8) is $R_{x}$-reversible.

(iii) If $5 a_{22}-3 b_{13}=0, a_{50} \neq 0, a_{22} \neq 0$. Imposing this condition, i.e., $b_{13}=\frac{5}{3} a_{22}$, we get

$$
\alpha_{11}=-\frac{385}{48} a_{22} a_{50} \neq 0 .
$$

Therefore system (8) is not orbitally $R_{x}$-reversible.

Now we study the orbital $R_{y}$-reversibility. Using Theorem 4 statement (a), a normal form of system (8) is

$$
\left(\begin{array}{c}
\dot{x} \\
\dot{y}
\end{array}\right)=\mathbf{F}_{7}+\left(\begin{array}{c}
y F\left(x, y^{2}\right) \\
G\left(x, y^{2}\right)
\end{array}\right)+\sum_{k \geq 1}\left(\mathbf{x}_{\widetilde{g}_{12+k}}+\bar{\eta}_{7+k} \mathbf{D}_{0}\right),
$$

where $\widetilde{g}_{12+k} \in \operatorname{Cor}\left(\widetilde{\ell}_{12+k}^{c}\right)$ and $\bar{\eta}_{7+k} \in \operatorname{Cor}\left(\bar{\ell}_{7+k}\right)$.

First, we are going to determine $\operatorname{Cor}\left(\ell_{8}\right)$ and $\operatorname{Cor}\left(\ell_{13}^{c}\right)$.

- $\quad \widetilde{\mathcal{P}}_{1}^{\mathbf{t}}=\{0\}$ and $\overline{\mathcal{P}}_{8}^{\mathbf{t}}=\operatorname{span}\left\{x^{4}, x y^{2}\right\}$, therefore $\operatorname{Cor}\left(\bar{\ell}_{8}\right)=\operatorname{span}\left\{x^{4}, x y^{2}\right\}$.

- $\bar{\Delta}_{6}=\operatorname{span}\left\{x^{3}, y^{2}\right\}$ and $\widetilde{\Delta}_{13}=\operatorname{span}\left\{x^{5} y, x^{2} y^{3}\right\}$. If we take $g(x, y)=c_{30} x^{3}+c_{02} y^{2} \in$ $\bar{\Delta}_{8}^{\mathrm{t}}$, then $\widetilde{\ell}_{13}^{\mathrm{c}}(g)=\left(6 a c_{30}-2 c_{02}\right) x^{5} y+\left(3 c_{30}-6 a c_{02}\right) x^{2} y^{3}$ and therefore $\operatorname{Cor}\left(\widetilde{\ell}_{13}^{\mathrm{c}}\right)=$ $\{0\}$.

So, $\bar{\eta}_{8}=\alpha_{8}^{(1)} x^{4}+\alpha_{8}^{(2)} x y^{2}$ and $\widetilde{g}_{13}=0$.

In this case, using the theory of normal forms, we obtain

$$
\alpha_{8}^{(1)}=\frac{1}{13}\left(5 a_{50}+b_{41}\right)=0, \quad \alpha_{8}^{(2)}=\frac{1}{3}\left(2 a_{22}+3 b_{13}\right)=0 .
$$

Imposing these conditions we obtain case a). Sufficient condition is previously demonstrated.

This completes the proof.

Example 3. Consider the differential system whose vector field is sum of two quasi-homogenous fields of degrees 13 and 14 , respectively, with respect to type $\mathbf{t}=(2,5)$

$$
\left(\begin{array}{c}
\dot{x} \\
\dot{y}
\end{array}\right)=\overbrace{\left(\begin{array}{c}
y^{3}+a_{51} x^{5} y \\
-x^{9}+b_{42} x^{4} y^{2}
\end{array}\right)}^{\mathbf{F}_{13}}+\overbrace{\left(\begin{array}{c}
a_{80} x^{8}+a_{32} x^{3} y^{2} \\
b_{71} x^{7} y+b_{23} x^{2} y^{3}
\end{array}\right)}^{\mathbf{F}_{14}} .
$$

We are interested in studying the orbital reversibility of system (9), whose origin is monodromic, in order to calculate degenerate centers.

First, we study the monodromy of system (9).

Proposition 7. The origin of system (9) is monodromic if, and only if, $\left(2 b_{42}-5 a_{51}\right)^{2}<40$.

Proof. The Hamiltonian function of the first quasi-homogeneous component of system (9) is

$$
h(x, y)=-\frac{1}{10} x^{10}+\frac{2 b_{42}-5 a_{51}}{20} x^{5} y^{2}-\frac{1}{4} y^{4}=-\frac{1}{10}\left[\left(x^{5}-\frac{2 b_{42}-5 a_{51}}{4} y^{2}\right)^{2}+\frac{1}{16}\left(40-\left(2 b_{42}-5 a_{51}\right)^{2}\right) y^{4}\right] .
$$

If $40-\left(2 b_{42}-5 a_{51}\right)^{2}>0, h(x, y)$ is negative-defined and then the origin of system (9) is monodromic.

If $40-\left(2 b_{42}-5 a_{51}\right)^{2}<0, h(x, y)$ has simple real factors. By [44] [Proposition 6] the origin of system (9) in this case is not monodromic. 
If $40-\left(2 b_{42}-5 a_{51}\right)^{2}=0$, i.e., $b_{42}=\frac{5}{2} a_{51}+\sigma \sqrt{10}$ with $\sigma= \pm 1$. In this case $h(x, y)$ has a real factor of multiplicity two. This factor is $x^{5}-\sigma \frac{\sqrt{10}}{2} y^{2}$.

Applying to system (9) the directional blow-up $x=\left(v+\sigma \sqrt[5]{\frac{\sqrt{10}}{2}}\right) u^{2}, y=u^{5}$ and the reparametrization in the time $d t=\frac{d T}{u^{13}}$ we obtain

$$
\begin{aligned}
d u / d T & =u\left[-2^{3 / 5} 5^{2 / 5}\left(\sigma \sqrt{10}+5 a_{51}\right)+\mathcal{O}(\|u, v\|)\right] \\
d v / d T & =-\frac{80^{4 / 5}}{800} \sqrt{10}\left[\left(2 b_{71}-5 a_{80}\right) \sqrt{10}+2\left(2 b_{23}-5 a_{32}\right) \sigma\right] u+\mathcal{O}\left(\|u, v\|^{2}\right)
\end{aligned}
$$

- If $\sigma \sqrt{10}+5 a_{51} \neq 0$ the Newton diagram of system (10) has even ordinates. Then by [44] [Theorem 2, item 3b] the origin of system (9) is not monodromic.

- If $a_{51}=-\frac{\sigma}{5} \sqrt{10}$ then $b_{42}=\frac{\sigma}{2} \sqrt{10}$ and system (10) is

$$
\begin{aligned}
d u / d T & =u\left[-\frac{80^{1 / 5}}{20}\left(b_{71} \sqrt{10} \sigma+2 b_{23}\right) u+\frac{1250^{1 / 5}}{2} v+\mathcal{O}\left(\|u, v\|^{2}\right)\right] \\
d v / d T & =-\frac{80^{4 / 5}}{800} \sqrt{10}\left[\left(2 b_{71}-5 a_{80}\right) \sqrt{10}+2\left(2 b_{23}-5 a_{32}\right) \sigma\right] u+\mathcal{O}\left(\|u, v\|^{2}\right) .
\end{aligned}
$$

(i) If $\left(2 b_{71}-5 a_{80}\right) \sqrt{10}+2\left(2 b_{23}-5 a_{32}\right) \sigma \neq 0$, the Newton diagram of system (11) has only one compact wedge whose associated vector field is

$$
\mathbf{F}_{1}=\left(\frac{1250^{1 / 5}}{2} u v,-\frac{80^{4 / 5}}{800} \sqrt{10}\left[\left(2 b_{71}-5 a_{80}\right) \sqrt{10}+2\left(2 b_{23}-5 a_{32}\right) \sigma\right] u\right) \in \mathcal{Q}_{1}^{(2,1)}
$$

and its Hamiltonian function is

$$
h_{4}(u, v)=-u\left(\frac{80^{4 / 5}}{400} \sqrt{10}\left[\left(2 b_{71}-5 a_{80}\right) \sqrt{10}+2\left(2 b_{23}-5 a_{32}\right) \sigma\right] u+\frac{1250^{1 / 5}}{2} v\right),
$$

that has a strong factor. By [44] [Theorem 2, item 2b], the origin of system (9) is not monodromic.

(ii) If $\left.b_{71}=\frac{\sqrt{10}}{20}\left(5 a_{80} \sqrt{10}+10 a_{32} \sigma-4 b_{23} \sigma\right)\right), v=0$ is an invariant axis of system (11), then the origin of system (9) is not monodromic.

Consequently the proof is complete.

In order to study the orbital reversibility of the family (9) whose origin is monodromic, i.e., $\left(2 b_{42}-5 a_{51}\right)^{2}<40$, we first have to calculate the possible reversibilities of the first component $\mathbf{F}_{13}$ (modulo a zero-degree change of variables).

Proposition 8. The quasi-homogeneous vector field $\mathbf{F}_{13}$ given in (9) is $R_{x}$-reversible if $a_{51}=$ $b_{42}=0$ and $\mathbf{F}_{13}$ is $R_{y}$-reversible for all $a_{51}, b_{42} \in \mathbb{R}$ and they are the only reversibilities of $\mathbf{F}_{13}$ (modulo a zero-degree change of variables).

Proof. For the type $\mathbf{t}=(2,5)$ we get $\mathcal{Q}_{0}^{\mathbf{t}}=\left\{\left(a_{10} x, b_{01} y\right)^{T}: a_{10}, b_{01} \in \mathbb{R}\right\}$, then $\left(\Phi_{0}\right)_{*} \mathbf{F}_{13}$ is $\mathrm{R}_{x}$ reversible if and only if $a_{51}=b_{42}=0$, and $\left(\Phi_{0}\right)_{*} \mathbf{F}_{13}$ is $\mathrm{R}_{y}$ reversible for all $\Phi_{0} \in \mathcal{Q}_{0}^{\mathrm{t}}$. Therefore, these two are the only reversibilities of $\mathbf{F}_{13}$ modulo a zero-degree change of variables.

Remark 2. As a consequence of the previous result we only have to analyze the orbital $R_{x^{-}}$ reversibility of system (9) for $a_{51}=b_{42}=0$ and the orbital $R_{y}$-reversibility for $a_{51}, b_{42} \in \mathbb{R}$.

Theorem 7. If the origin of system (9) is monodromic then system is orbitally reversible if, and only if, one of the following conditions holds:

(a) $a_{80}=a_{32}=b_{71}=b_{23}=0\left(R_{y}\right.$-reversible case $)$. 
(b) $5 a_{51}+2 b_{42}=3 a_{80}-a_{51} a_{32}=3 b_{71}+8 a_{51} a_{32}=b_{23}+a_{32}=0$ (particular case of Hamiltonian)

(c) $a_{51}=b_{42}=a_{32}=b_{23}=0\left(R_{x}\right.$-reversible case $)$.

Proof. By Remark 2 we have only to study the orbital $R_{x}$ - or $R_{y}$-reversibility.

First we study the orbital $R_{x}$-reversibility. From Proposition 8 it must be $a_{51}=b_{42}=0$. In this case the first quasi-homogeneous component is a Hamiltonian vector field $\mathbf{F}_{13}=\mathbf{X}_{h}$, where $h=-x^{10} / 10-y^{4} / 4$ with simple factor in $\mathbb{C}[x, y]$. Thus, by Lemma 9, we get $\operatorname{Ker}\left(\widetilde{\ell}_{r+k+|\mathbf{t}|}^{c}\right)=\{0\}$ and we can apply Theorem 2 .

Applying Theorem 2 statement (a). a normal form of system (9) is

$$
\left(\begin{array}{c}
\dot{x} \\
\dot{y}
\end{array}\right)=\mathbf{x}_{h}+\left(\begin{array}{c}
F\left(x^{2}, y\right) \\
x G\left(x^{2}, y\right)
\end{array}\right)+\sum_{k \geq 1}\left(\mathbf{x}_{\tilde{g}_{20+k}}+\bar{\eta}_{13+k} \mathbf{D}_{0}\right)
$$

where $\widetilde{g}_{20+k} \in \operatorname{Cor}\left(\widetilde{\ell}_{20+k}^{c}\right)$ and $\bar{\eta}_{13+k} \in \operatorname{Cor}\left(\bar{\ell}_{13+k}\right)$. So, by Theorem 2 item (b), system (9) is orbitally $R_{x}$-reversible if, and only if, $\widetilde{g}_{20+k}=\bar{\eta}_{13+k}=0$ for $k \in \mathbb{N}$. Using Lemma 10 stated below and Proposition 1 we obtain $\operatorname{Cor}\left(\bar{\ell}_{14}\right)=\operatorname{span}\left\{x^{2} y^{2}\right\}, \operatorname{Cor}\left(\bar{\ell}_{15}\right)=\{0\}$, $\operatorname{Cor}\left(\bar{\ell}_{16}\right)=\operatorname{span}\left\{x^{8}\right\}$ and $\operatorname{Cor}\left(\bar{\ell}_{17}\right)=\operatorname{span}\left\{x^{6} y\right\}$. So $\bar{\eta}_{14}=\alpha_{14} x^{2} y^{2}, \bar{\eta}_{15}=0, \bar{\eta}_{16}=$ $\alpha_{16} x^{8}, \bar{\eta}_{17}=\alpha_{17} x^{6} y$. Analogously, applying Lemma 11 stated below and Proposition 4, we have $\operatorname{Cor}\left(\widetilde{\ell}_{21}^{c}\right)=\operatorname{Cor}\left(\widetilde{\ell}_{22}^{c}\right)=\operatorname{Cor}\left(\widetilde{\ell}_{23}^{c}\right)=\{0\}$ and $\operatorname{Cor}\left(\widetilde{\ell}_{24}^{c}\right)=\operatorname{span}\left\{x^{7} y^{2}\right\}$. So $\widetilde{g}_{21}=0, \widetilde{g}_{22}=0, \widetilde{g}_{23}=0, \widetilde{g}_{24}=\beta_{24} x^{7} y^{2}$.

The value obtained at order 14 is $\alpha_{14}=3\left(a_{32}+b_{23}\right) / 20$. If we vanish this constant assuming $b_{23}=-a_{32}$ the next constant is $\alpha_{16}=3 a_{32}\left(8 a_{80}+b_{71}\right)\left(3 a_{80}-b_{71}\right) / 220$. Hence we have three possibilities $a_{32}=0,8 a_{80}+b_{71}=0$ and $3 a_{80}-b_{71}=0$.

If $a_{32}=0$, we get the case (c).

If $8 a_{80}+b_{71}=0$ and $a_{32} \neq 0$ then taking $b_{71}=-8 a_{80}$, the next constants are

$$
\beta_{24}=\frac{34}{123} a_{32}^{3} a_{80}, \quad \alpha_{17}=\frac{308}{405} a_{32}^{3} a_{80} .
$$

Then $a_{80}=0$ and therefore $b_{71}=0$ and we obtain a particular case of case (b).

If $3 a_{80}-b_{71}=0$, with $a_{32}\left(8 a_{80}+b_{71}\right) \neq 0$, taking $b_{71}=3 a_{80}$, the next constants are

$$
\beta_{24}=-\frac{17}{324} a_{32}^{3} a_{80}, \quad \alpha_{17}=-\frac{77}{270} a_{32}^{3} a_{80},
$$

that cannot be canceled since $a_{32} \neq 0$ and, if $a_{80}=0$, then $b_{71}=0$ giving a contradiction.

Second, we study the orbital $R_{y}$-reversibility. From Proposition 8 we consider $a_{51}, b_{42}$ arbitrary. By Lemma 11 stated below, we have that $\operatorname{Ker}\left(\ell_{20+k}^{c}\right)=\{0\}$ for all $k \in \mathbb{N}$ then $\operatorname{Ker}\left(\widetilde{\ell}_{r+k+|\mathbf{t}|}^{c}\right)=\{0\}$ (see proof of Proposition 4) and therefore the hypothesis of the Theorem 4 are fulfilled.

Applying Theorem 4 item (a), a normal form of system (9) is

$$
\left(\begin{array}{c}
\dot{x} \\
\dot{y}
\end{array}\right)=\mathbf{F}_{13}+\left(\begin{array}{c}
y F\left(x, y^{2}\right) \\
G\left(x, y^{2}\right)
\end{array}\right)+\sum_{k \geq 1} \mathbf{x}_{\widetilde{g}_{20+k}}+\bar{\eta}_{13+k} \mathbf{D}_{0}
$$

where $\widetilde{g}_{20+k} \in \operatorname{Cor}\left(\widetilde{\ell}_{20+k}^{c}\right)$ and $\bar{\eta}_{13+k} \in \operatorname{Cor}\left(\bar{\ell}_{13+k}\right)$. So, by Theorem 4 item (b), system (9) is orbitally $R_{y}$-reversible if, and only if, $\widetilde{g}_{20+k}=\bar{\eta}_{13+k}=0$ for $k \in \mathbb{N}$ (in this case with distinguished variable $y$ ). Using Lemma 10 stated below and Proposition 1 we have $\operatorname{Cor}\left(\bar{\ell}_{14}\right)=\operatorname{span}\left\{x^{7}, x^{2} y^{2}\right\}, \operatorname{Cor}\left(\bar{\ell}_{15}\right)=\{0\}$ and $\operatorname{Cor}\left(\bar{\ell}_{16}\right)=\operatorname{span}\left\{x^{8}, x^{3} y^{2}\right\}$. So $\bar{\eta}_{14}=$ $\alpha_{14}^{(1)} x^{7}+\alpha_{14}^{(2)} x^{2} y^{2}, \bar{\eta}_{15}=0, \bar{\eta}_{16}=\alpha_{16}^{(1)} x^{8}+\alpha_{16}^{(2)} x^{3} y^{2}$. Analogously, using Lemma 11 stated 
below and Proposition 4, we obtain $\operatorname{Cor}\left(\widetilde{\ell}_{21}^{c}\right)=\operatorname{span}\left\{x^{8} y\right\}, \operatorname{Cor}\left(\widetilde{\ell}_{22}^{c}\right)=\operatorname{Cor}\left(\widetilde{\ell}_{23}^{c}\right)=\{0\}$. So $\widetilde{g}_{21}=\beta_{21} x^{8} y, \widetilde{g}_{22}=0, \widetilde{g}_{23}=0$.

The values obtained at order 14 are

$$
\begin{aligned}
& \beta_{21}=\frac{1}{441}\left(42 b_{71}-105 a_{80}+8 b_{42} b_{23}-20 b_{42} a_{32}-22 a_{51} b_{23}+55 a_{51} a_{32}\right), \\
& \alpha_{14}^{(1)}=\frac{1}{420}\left(21 b_{71}+168 a_{80}-25 a_{32} a_{51}-10 a_{32} b_{42}+10 a_{51} b_{23}+4 b_{23} b_{42}\right), \\
& \alpha_{14}^{(2)}=\frac{3}{20}\left(a_{32}+b_{23}\right) .
\end{aligned}
$$

If we vanish these constants, we get

$$
a_{80}=\frac{1}{3} a_{51} a_{32}, \quad b_{71}=-a_{51} a_{32}+\frac{2}{3} b_{42} a_{32}, \quad b_{23}=-a_{32} .
$$

Imposing these equalities the second condition is

$$
\begin{aligned}
\alpha_{16}^{(1)} & =-\frac{23}{270} \frac{\left(5 a_{51}+2 b_{42}\right)\left(-13 b_{42}+25 a_{51}\right) a_{32}^{3}}{-529+52 b_{42}^{2}-269 b_{42} a_{51}+325 a_{51}^{2}} \\
\alpha_{16}^{(2)} & =\frac{1058}{135} \frac{a_{32}^{3}\left(5 a_{51}+2 b_{42}\right)}{-529+52 b_{42}^{2}-269 b_{42} a_{51}+325 a_{51}^{2}} .
\end{aligned}
$$

The vanishing of these two constants gives two possibilities, $a_{32}=0$ or $5 a_{51}+2 b_{42}=0$. The first one corresponds to case (a) and the second one to case (b), respectively.

Now we see the sufficiency.

(a) In this case the system is $R_{y}$-reversible.

(b) In this case the system (9) is Hamiltonian, i.e., $\mathbf{F}=\mathbf{X}_{H}$ with a Hamiltonian function $H=h+h_{21}$, where $h(x, y)=-x^{10} / 10-a_{51} x^{5} y^{2} / 2-y^{4} / 4 \in \mathcal{P}_{20}^{\mathrm{t}}$ and $h_{21}(x, y)=$ $-a_{51} a_{32} x^{8} y / 3-a_{32} x^{3} y^{3} / 3 \in \mathcal{P}_{21}^{\mathrm{t}}$. Taking into account that in the conservative case, i.e., $5 a_{51}+2 b_{42}=0$, we have that $\ell_{20+k}^{c}=\operatorname{Proy}_{\Delta_{20+k}}\left(\left.\ell_{20+k}\right|_{\Delta_{13+k}}\right)$, by [43] [Theorem 4] $H$ is conjugated to a polynomial vector field $G=h+\sum_{k=1}^{16} g_{20+k}$ with $g_{20+k} \in \operatorname{Cor}\left(\ell_{20+k}^{c}\right)$. By Lemma 11 stated below, $H$ is conjugated to $G=$ $h+\beta_{21} x^{8} y+\beta_{22} x^{6} y^{2}+\beta_{24} x^{7} y^{2}+\beta_{26} x^{8} y^{2}$.

If we apply the change of variables $x=u, y=v-\frac{1}{3} a_{32} u^{3}$, the function $H$ is transformed into $\widetilde{H}=h+\widetilde{h}_{22}+\widetilde{h}_{23}+\widetilde{h}_{24}$, where $\widetilde{h}_{22}(u, v)=a_{51} a_{32}^{2} u^{11} / 18+a_{32}^{2} u^{6} v^{2} / 6 \in$ $\mathcal{P}_{22}^{\mathrm{t}}, \widetilde{h}_{23}(u, v)=-2 a_{32}^{3} u^{9} v / 27 \in \mathcal{P}_{23}^{\mathrm{t}}$ and $\widetilde{h}_{24}=a_{32}^{4} u^{12} / 108 \in \mathcal{P}_{24}^{\mathrm{t}}$. Therefore $H$ is conjugated to $G=h+\beta_{22} x^{6} y^{2}+\beta_{24} x^{7} y^{2}+\beta_{26} x^{8} y^{2}$, because the quasi-homogeneous term of degree 21 in $\widetilde{H}$ is null, i.e., $\beta_{21}=0$. By Proposition 5, system (9) is orbitally equivalent to system $(\dot{x}, \dot{y})^{\mathbf{t}}=\mathbf{X}_{G}$ which is $R_{y}$-reversible and, consequently, system (9) is orbitally reversible.

(c) In this case the vector field is $R_{x}$-reversible.

This completes the proof.

Appendix for Example 2

Here we present two technical lemmas used in the study of Example 2.

Lemma 10. The first four subspaces $\operatorname{Cor}\left(\ell_{13+k}\right), k=1,2,3,4$, where $\ell_{13+k}$ is the Lie-derivative of $\mathbf{F}_{13}$, the first quasi-homogeneous term of system (9) are:

$\operatorname{Cor}\left(\ell_{14}\right)=\operatorname{span}\left\{x^{7}, x^{2} y^{2}\right\}, \operatorname{Cor}\left(\ell_{15}\right)=\operatorname{span}\left\{x^{5} y\right\}, \operatorname{Cor}\left(\ell_{16}\right)=\operatorname{span}\left\{x^{8}, x^{3} y^{2}\right\}$ and $\operatorname{Cor}\left(\ell_{17}\right)=\operatorname{span}\left\{x y^{3}\right\}$ if $a_{51} \neq 0, \operatorname{Cor}\left(\ell_{17}\right)=\operatorname{span}\left\{x^{6} y\right\}$ if $a_{51}=0$.

\section{Proof.}

- $\quad$ Case $k=1$, we have $\mathcal{P}_{1}^{\mathbf{t}}=\{0\}$ and $\mathcal{P}_{14}^{\mathbf{t}}=\operatorname{span}\left\{x^{7}, x^{2} y^{2}\right\}$. Therefore $\operatorname{Cor}\left(\ell_{14}\right)=$ $\operatorname{span}\left\{x^{7}, x^{2} y^{2}\right\}$. 
- $\quad$ Case $k=2$, we have $\mathcal{P}_{2}^{\mathrm{t}}=\operatorname{span}\{x\}$ and $\mathcal{P}_{15}^{\mathrm{t}}=\operatorname{span}\left\{x^{5} y \cdot y^{3}\right\}$. If $p_{2}=u_{0} x$ then $\nabla p_{2} \cdot \mathbf{F}_{13}=u_{0} y^{3}+u_{0} a_{51} x^{5} y$. Therefore we can choose $\operatorname{Cor}\left(\ell_{15}\right)=\operatorname{span}\left\{x^{5} y\right\}$.

- $\quad$ Case $k=3$, we have $\mathcal{P}_{3}^{\mathbf{t}}=\{0\}$ and $\mathcal{P}_{16}^{\mathbf{t}}=\operatorname{span}\left\{x^{8}, x^{3} y^{2}\right\}$. Therefore $\operatorname{Cor}\left(\ell_{16}\right)=$ $\operatorname{span}\left\{x^{8}, x^{3} y^{2}\right\}$.

- $\quad$ Case $k=4$, we have $\mathcal{P}_{4}^{\mathrm{t}}=\operatorname{span}\left\{x^{2}\right\}$ and $\mathcal{P}_{17}^{\mathrm{t}}=\operatorname{span}\left\{x^{6} y, x y^{3}\right\}$. Therefore we can choose $\operatorname{Cor}\left(\ell_{17}\right)=\operatorname{span}\left\{x y^{3}\right\}$ if $a_{51} \neq 0$ and $\operatorname{Cor}\left(\ell_{17}\right)=\operatorname{span}\left\{x^{6} y\right\}$ if $a_{51}=0$.

Lemma 11. If the origin of system (9) is monodromic the following conditions are verified: $\operatorname{Ker}\left(\ell_{20+k}^{c}\right)=\{0\}$ for all $k \in \mathbb{N}, \operatorname{Cor}\left(\ell_{20+k}^{c}\right)=\{0\}$ for $k \in \mathbb{N}, k \notin\{1,2,4,6\}$ and $\operatorname{Cor}\left(\ell_{21}^{c}\right)=$ $\operatorname{span}\left\{x^{8} y\right\}, \operatorname{Cor}\left(\ell_{22}^{c}\right)=\operatorname{span}\left\{x^{6} y^{2}\right\}, \operatorname{Cor}\left(\ell_{24}^{c}\right)=\operatorname{span}\left\{x^{7} y^{2}\right\}, \operatorname{Cor}\left(\ell_{26}^{c}\right)=\operatorname{span}\left\{x^{8} y^{2}\right\}$.

Proof. Rename $a_{51}=2 d_{41}-2 c_{52}, b_{42}=5 d_{41}+5 c_{52}$. We have that the first quasi-homogeneous component is $\mathbf{F}_{13}=\mathbf{X}_{-\frac{1}{10} x^{10}+c_{52} x^{5} y^{2}-\frac{1}{4} y^{4}}+d_{41} x^{4} y \mathbf{D}_{0}$. The inverse change is $c_{52}=\frac{2 b_{42}-5 a_{51}}{20}$, $d_{41}=\frac{5 a_{51}+2 b_{42}}{20}$. Taking into account that the origin of system (9) is monodromic, by Proposition 7 we have that $\left(2 b_{42}-5 a_{51}\right)^{2}<40$, which implies $c_{52}^{2}<1 / 10$. Now we study the subspaces $\operatorname{Ker}\left(\ell_{20+k}^{c}\right)$ and $\operatorname{Cor}\left(\ell_{20+k}^{c}\right)$ for $k \in \mathbb{N}$.

- Case $k=1$. We have $\Delta_{8}=\operatorname{span}\left\{x^{4}\right\}$ and $\Delta_{21}=\operatorname{span}\left\{x^{8} y, x^{3} y^{3}\right\}$. Moreover if $p_{8}=u_{40} x^{4} \in \Delta_{8}$ then $\ell_{21}^{c}\left(p_{8}\right)=\frac{8}{21}\left(-21 c_{52}+d_{41}\right) u_{0} x^{8} y+4 u_{0} x^{3} y^{3}$ and therefore $\operatorname{Ker}\left(\ell_{21}^{\mathrm{c}}\right)=\{0\}$ and $\operatorname{Cor}\left(\ell_{21}^{\mathrm{c}}\right)=\operatorname{span}\left\{x^{8} y\right\}$.

- Case $k=2 l-1, l \geq 2$. We have $\Delta_{2(l+3)}=\operatorname{span}\left\{x^{l+3}, x^{l-2} y^{2}\right\}$ and $\Delta_{2(l+9)+1}=$ $\operatorname{span}\left\{x^{l+7} y, x^{l+2} y^{3}\right\}$. If $p_{2(l+3)}=u_{0} x^{l+3}+u_{1} x^{l-2} y^{2}$ then

$$
\begin{aligned}
\ell_{2(l+9)+1}^{\mathrm{c}}\left(p_{2(l+3)}\right)= & \frac{l+3}{5(2 l+19)}\left\{-2\left[\left(5(2 l+19) c_{52}-5(2 l-1) d_{41}\right) u_{0}+(2 l+19) u_{1}\right] x^{l+7} y\right. \\
& \left.+5\left[(2 l+19) u_{0}+2\left((2 l+19) c_{52}+(2 l-1) d_{41}\right) u_{1}\right] x^{l+2} y^{3}\right\}
\end{aligned}
$$

Therefore $\operatorname{Ker}\left(\ell_{2(l+9)+1}^{c}\right)=\{0\}$ and $\operatorname{Cor}\left(\ell_{2(l+9)+1}^{c}\right)=\{0\}$ if $0 \neq(2 l+19)^{2} c_{52}^{2}-$ $(2 l-1)^{2} d_{41}^{2}-\frac{(2 l+19)^{2}}{10}$ and, as $c_{52}^{2}<\frac{1}{10}$, this condition is satisfied.

- $\quad$ Case $k=2$. We have that $\Delta_{9}=\operatorname{span}\left\{x^{2} y\right\}$ and $\Delta_{22}=\operatorname{span}\left\{x^{11}, x^{6} y^{2}\right\}$. If $p_{9}=u_{0} x^{2} y$ then $\ell_{22}^{c}\left(p_{9}\right)=-\frac{9}{5} u_{0} x^{11}+\frac{9}{11}\left(11 c_{52}+d_{41}\right) u_{0} x^{6} y^{2}$. Therefore $\operatorname{Ker}\left(\ell_{22}^{c}\right)=\{0\}$ and $\operatorname{Cor}\left(\ell_{22}^{c}\right)=\operatorname{span}\left\{x^{6} y^{2}\right\}$.

- Case $k=4$. We have that $\Delta_{11}=\operatorname{span}\left\{x^{3} y\right\}$ and $\Delta_{24}=\operatorname{span}\left\{x^{12}, x^{7} y^{2}\right\}$. If $p_{11}=$ $u_{0} x^{3} y$ then $\ell_{24}^{c}\left(p_{11}\right)=-\frac{11}{5} u_{31} x^{12}+\frac{11}{120} u_{31}\left(-25 a_{51}+14 b_{42}\right) x^{7} y^{2}$. Therefore we have $\operatorname{Ker}\left(\ell_{24}^{\mathrm{c}}\right)=\{0\}$ and $\operatorname{Cor}\left(\ell_{24}^{\mathrm{c}}\right)=\operatorname{span}\left\{x^{7} y^{2}\right\}$.

- Case $k=6$. We have that $\Delta_{13}=\operatorname{span}\left\{x^{4} y\right\}$ and $\Delta_{26}=\operatorname{span}\left\{x^{13}, x^{8} y^{2}\right\}$. If $p_{13}=$ $u_{0} x^{4} y$ then $\ell_{26}^{c}\left(p_{13}\right)=-\frac{13}{5} u_{0} x^{13}+u_{0}\left(13 c_{52}+3 d_{41}\right) x^{8} y^{2}$. Therefore we have $\operatorname{Ker}\left(\ell_{26}^{c}\right)$ $=\{0\}$ and $\operatorname{Cor}\left(\ell_{26}^{c}\right)=\operatorname{span}\left\{x^{8} y^{2}\right\}$.

- Case $k=2 l, l \geq 4$. We have $\Delta_{2(l+3)+1}=\operatorname{span}\left\{x^{l+1} y, x^{l-4} y^{3}\right\}$ and $\Delta_{2(l+10)}=$ $\operatorname{span}\left\{x^{l+10}, x^{l+5} y^{2}\right\}$. If $p_{2(l+3)+1}=u_{0} x^{l+1} y+u_{1} x^{l-4} y^{3}$ then

$$
\begin{aligned}
& \ell_{2(l+10)}^{c}\left(p_{2(l+3)+1}\right)=\frac{2 l+7}{5(l+10)}\left\{\left[-10(l+10) u_{0}+2\left((l+10) c_{52}+l d_{41}\right) u_{1}\right] x^{l+10}\right. \\
& \left.+\left[5\left((l+10) c_{52}+l d_{41}\right) u_{0}+\left[\left(20 c_{52}\left((l+10) c_{52}+l d_{41}\right)-l-10\right)\right] u_{1}\right] x^{l+5} y^{2}\right\}
\end{aligned}
$$




$$
\begin{aligned}
& \text { Therefore } \operatorname{Ker}\left(\ell_{2(l+10)}^{c}\right)=\{0\} \text { and } \operatorname{Cor}\left(\ell_{2(l+10)}^{c}\right)=\{0\} \text { if it is satisfied }(l+10)^{2} c_{52}^{2}- \\
& d_{41}^{2}-\frac{(l+10)^{2}}{10} \neq 0 \text {. But since } c_{52}^{2}<1 / 10 \text {, this condition is verified. }
\end{aligned}
$$

Author Contributions: A.A., C.G. and J.G. did the formal analysis, provided the methodology, and did the writing-review and editing. All authors have read and agreed to the published version of the manuscript.

Funding: The first and second authors are partially supported by Ministerio de Ciencia, Innovación y Universidades/ FEDER grant number PGC2018-096265-B-I00 and by the Consejería de Educación y Ciencia de la Junta de Andalucía (projects P12-FQM-1658, FQM-276, UHU-1260150). The third author is partially supported by a MINECO/ FEDER grant number MTM2017-84383-P and an AGAUR (Generalitat de Catalunya) grant number 2017SGR-1276.

Data Availability Statement: Data sharing not applicable. No new data were created or analyzed in this study. Data sharing is not applicable to this article.

Acknowledgments: The authors are grateful to the referees for their valuable comments and suggestions to improve this paper.

Conflicts of Interest: The authors declare no conflict of interest.

\section{References}

1. Lamb, J.S.W.; Roberts, J.A.G. Time-reversal symmetry in dynamical systems: A survey. Phys. Sect. D 1998, 112, 1-39. [CrossRef]

2. Roberts, J.A.G.; Quispel, G.R.W. Chaos and time-reversal symmetry orden and chaos in reversible dynamical systems. Phys. Rep. 1992, 216, 63-177. [CrossRef]

3. Matveyev, M.V. Reversible systems with first integrals. Physica D 1998, 112, 148-157. [CrossRef]

4. Tkhai, V.N. The reversibility of mechanical systems. J. Appl. Math. Mech. 1991, 55, 461-469. [CrossRef]

5. Han, M.; Petek, T.; Romanovski, V.G. Reversibility in polynomial systems of ODE's. Appl. Math. Comput. $2018,338,55-71$. [CrossRef]

6. Romanovski, V.G. Time-reversibility in 2-dimensional systems. Open Syst. Inf. Dyn. 2008, 15, 359-370. [CrossRef]

7. Montgomery, D.; Zippin, L. Topological Transformations Groups; Interscience: New York, NY, USA, 1955.

8. Devaney, R.L. Reversibility, homoclinic points, and the Hnon map. In Dynamical Systems Approaches to Nonlinear Problems in Systems and Circuits, Henniker, NH, 1986; SIAM: Philadelphia, PA, USA, 1988; pp. 3-14.

9. Giné, J. The center problem for a linear center perturbed by homogeneous polynomials. Acta Math. Sin. (Engl. Ser.) 2006, 22, 1613-1620. [CrossRef]

10. Giné, J. The nondegenerate center problem and the inverse integrating factor. Bull. Sci. Math. 2006, 130, 152-161. [CrossRef]

11. Mazzi, L.; Sabatini, M. A characterization of centres via first integrals. J. Differ. Equ. 1988, 76, 222-237. [CrossRef]

12. Algaba, A.; Gamero, E.; García, C. The center problem. A view from the normal form theory. J. Math. Anal. Appl. 2016, 434, 680-697. [CrossRef]

13. Teixeira, M.A.; Yang, J. The center-focus problem and reversibility. J. Differ. Equ. 2001, 174, 237-251. [CrossRef]

14. Zoladek, H.; Llibre, J. The Poincar center problem. J. Dyn. Control Syst. 2008, 14, 505-535. [CrossRef]

15. Bibikov, Y.N. Local Theory of Nonlinear Analytic Ordinary Differential Equations. Lecture Notes in Mathematics; Springer: Berlin/ Heidelberg, Germany, 1982; Volume 702.

16. Bruno, A.D. Local Methods in Nonlinear Differential Equations; Springer: Berlin/Heidelberg, Germany, 1989.

17. Arnold, V.I. Geometrical Methods in the Theory of Differential Equations; Springer: Berlin/Heidelberg, Germany, 1982.

18. Poincaré, H. Sur l'intégration algébrique des équations différentielles du premier ordre et du premier degré I. Rend. Circ. Mat. Palermo 1897, 5, 161-191; Rend. Circ. Mat. Palermo 1879, 11, 193-234. [CrossRef]

19. Lyapunov, A.M. Stability of motion. In Mathematics in Science and Engineering; Academic Press: New York, NY, USA; London, UK, 1966; Volume 30.

20. Sadovskii, A.P. Problem of distinguishing a center and a focus for a system with a nonvanishing linear part. Differ. Uravn. 1976, 12, 1238-1246. (Translated).

21. Arnold, V.I.; Il'yashenko, Y.S. Enciclopaedia Math. Sci. Dynamical System 1; Springer: Berlin/Heidelberg, Germany, 1988.

22. Moussu, R. Symétrie et forme normale des centres et foyers dégénérés. Ergod. Theory Dyn. Syst. 1982, 2, 241-251. [CrossRef]

23. Berthier, M.; Moussu, R. Rversibilit et classification des centres nilpotents. J Ann. Inst. Fourier 1994, 44, 465-494. [CrossRef]

24. Algaba, A.; García, C.; Giné, J. Analytic integrability for some degenerate planar vector fields. J. Differ. Equ. 2014, 257, 549-565. [CrossRef]

25. Giné, J.; Maza, S. The reversibility and the center problem. Nonlinear Anal. 2011, 74, 695-704. [CrossRef] 
26. Giné, J.; Peralta-Salas, D. Existence of inverse integrating factors and Lie symmetries for degenerate planar centers. J. Differ. Equ. 2012, 252, 344-357. [CrossRef]

27. Giné, J. Sufficient conditions for a center at a completely degenerate critical point. Int. J. Bifurc. Chaos Appl. Sci. Eng. 2002, 12, 1659-1666. [CrossRef]

28. Giné, J. On the degenerate center problem. Int. J. Bifurc. Chaos Appl. Sci. Eng. 2011, 22, 1383-1392. [CrossRef]

29. García, I.A.; Giacomini, H.; Giné, J.; Llibre, J. Analytic nilpotent centers as limits of nondegenerate centers revisited. J. Math. Anal. Appl. 2016, 441, 893-899. [CrossRef]

30. Christopher, C.; Schlomiuk, D. On general algebraic mechanisms for producing centers in polynomial differential systems. J. Fixed Point Theory Appl. 2008, 3, 331-351. [CrossRef]

31. Giné, J.; Llibre, J. On the mechanisms for producing linear type centers in polynomial differential systems. Mosc. Math. J. 2018, 18, 409-420. [CrossRef]

32. Algaba, A.; Gamero, E.; García, C. The reversibility problem for quasi-homogeneous dynamical systems. Discret. Contin. Dyn. Syst. 2013, 33, 3225-3236. [CrossRef]

33. Algaba, A.; García, C.; Teixeira, M.A. Reversibility and quasi-homogeneous normal forms of vector fields. Nonlinear Anal. 2010, 73, 510-525. [CrossRef]

34. Lamb, J.S.W.; Roberts, J.A.G.; Capel, H.W. Condition for local (reversing) symmetries in dynamical systems. Physica A 1993, 197, 379-422. [CrossRef]

35. Algaba, A.; Checa, I.; García, C.; Gamero, E. On orbital-reversibility for a class of planar dynamical systems. Commun. Nonlinear Sci. Numer. Simul. 2015, 20, 229-239. [CrossRef]

36. Algaba, A.; García, C.; Giné, J. Center conditions of a particular polynomial differential system with a nilpotent singularity. J. Math. Anal. Appl. 2020, 483, 123639. [CrossRef]

37. Chow, S.N.; Hale, J.K. Methods of Bifurcation Theory; Springer: New York, NY, USA, 1982.

38. Algaba, A.; Gamero, E.; García, C. The integrability problem for a class of planar systems. Nonlinearity 2009, 22, 395-420. [CrossRef]

39. Algaba, A.; Freire, E.; Gamero, E.; García, C. Quasi-homogeneous normal forms. J. Comput. Appl. Math. 2003, 150, 193-216. [CrossRef]

40. Algaba, A.; García, C.; Giné, J. Analytic integrability around a nilpotent singularity. J. Differ. Equ. 2019, 267, 443-467. [CrossRef]

41. Giné, J. Liénard equation and its generalizations. Int. J. Bifurc. Chaos Appl. Sci. Eng. 2017, 27, 1750081. [CrossRef]

42. Liénard, A. Étude des oscillations entretenues. Rev. Gén. l'Électicité 1928, 23, 946-954.

43. Algaba, A.; García, C.; Reyes, M. Quasi-homogeneous linearization of degenerate vector fields. J. Math. Anal. Appl. 2020, 483, 123635. [CrossRef]

44. Algaba, A.; García, C.; Reyes, M. A new algorithm for determining the monodromy of a planar differential system. J. Appl. Math. Comput. 2014, 237, 419-429. [CrossRef] 